\title{
Interactions between Nodal and Wnt signalling Drive Robust Symmetry-Breaking and Axial Organisation in Gastruloids (Embryonic Organoids)
}

\author{
Turner D.A.. ${ }^{*}$, Glodowski C.R. ${ }^{1}$, Alonso-Crisostomo L. ${ }^{1}$, Baillie-Johnson P. ${ }^{1}$, Hayward P.C. ${ }^{1}$, \\ Collignon J. ${ }^{2}$, Gustavsen, C. ${ }^{3}$, Serup P. ${ }^{3}$, Schröter C. ${ }^{1,4}$, and Martinez Arias A. ${ }^{* 1}$ \\ ${ }^{1}$ Department of Genetics, University of Cambridge, Cambridge, CB2 3EH, United Kingdom \\ ${ }^{2}$ Universit Paris-Diderot, CNRS, Institut Jacques Monod, UMR 7592, Development and Neurobiology Programme, \\ F-75013 Paris, France \\ ${ }^{3}$ Danish Stem Cell Center, University of Copenhagen, DK-2200 Copenhagen, Denmark \\ ${ }^{4}$ Max Planck Institute of Molecular Physiology, Dortmund, Germany
}

May 13, 2016

\begin{abstract}
Generation of asymmetry within the early embryo is a critical step in the establishment of the three body axes, providing a reference for the patterning of the organism. To study the establishment of asymmetry and the development of the anteroposterior axis (AP) in culture, we utilised our 'Gastruloid' model system. Gastruloids, highly reproducible embryonic organoids formed from aggregates of mouse embryonic stem cells, display symmetry-breaking, polarised gene expression and axial development, mirroring the processes on a time-scale similar to that of the mouse embyro. Using Gastruloids formed from mouse ESCs containing reporters for Wnt, FGF and Nodal signalling, we were able to quantitatively assess the contribution of these signalling pathways to the establishment of asymmetry through single time-point and live-cell fluorescence microscopy.

During the first $24-48 \mathrm{~h}$ of culture, interactions between the Wnt/ $\beta$-Catenin and Nodal/TGF $\beta$ signalling pathways promote the initial symmetry-breaking event, manifested through polarised Brachyury $(\mathrm{T} / \mathrm{Bra})$ expression. Neither BMP nor FGF signalling is required for the establishment of asymmetry, however Wnt signalling is essential for the amplification and stability of the initial patterning event. Additionally, low, endogenous levels of FGF (24-48h) has a role in the amplification of the established pattern at later time-points.

Our results confirm that Gastruloids behave like epiblast cells in the embryo, leading us to translate the processes and signalling involved in pattern formation of Gastruloids in culture to the development of the embryo, firmly establishing Gastruloids as a highly reproducible, robust model system for studying cell fate decisions and early pattern formation in culture.
\end{abstract}

Key Words Gastruloids, mouse embryonic stem cells, symmetry-breaking, Wnt/ $\beta$-Catenin, Nodal/TGF $\beta$, embryonic organoids

\section{Introduction}

The emergence of the anteroposterior (AP) axis during the early stages of animal development is a fundamental patterning event that guides the spatial organisation of tissues and organs during embryogenesis. Comparative studies reveal that, although this process differs from one organism to another, in all cases it involves a symmetry-breaking event within a molecular or cellular isotropic system that results in the asymmetric localisation of signalling centres that will drive subsequent patterning events. Dipteran (e.g Drosophila) and amniote (e.g chicken and mouse) em- bryos provide extreme examples of the strategies associated with these processes. In Drosophila, the symmetry is broken within a single cell, the oocyte, which acquires both the AP and dorsoventral (DV) axes through well characterised processes of RNA and protein localisation that then serve as references for the rapid patterning of the embryo after fertilisation $[1,2]$. On the other hand, in birds and mammals the process occurs in the developing embryo, within a multicellular system, and leads to the assignation of fates to cell populations [3-7].

Efforts to understand the mechanisms that pattern early embryos have commonly relied on genetic

*to whom correspondence is to be addressed: ama11@cam.ac.uk, dat40@cam.ac.uk 

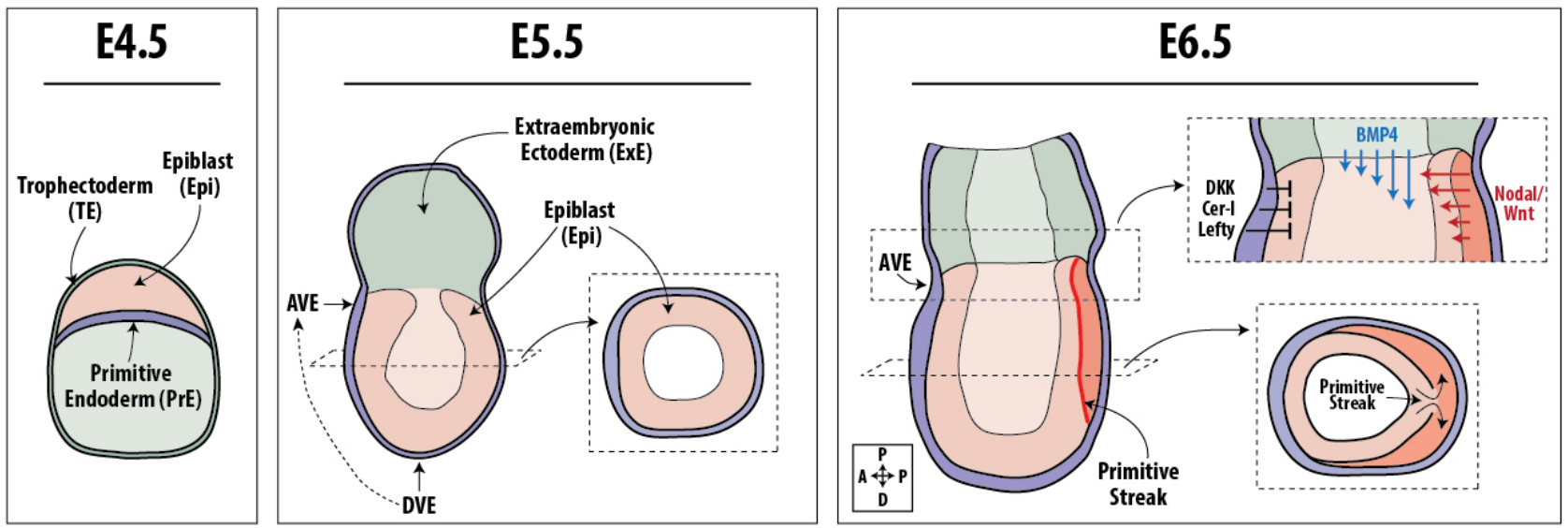

Figure 1: Establishment of the Anteroposterior Axis in the Mouse. At E4.5, following several rounds of cell division and the segregation of the extraembryonic tissues (the Primitive Endoderm (PrE) and the Trophoectoderm (TE)), the embryo is represented in the blastocyst, a mass of equivalent cells that undergoes a process of epithelialisation. By stage E5.5 the embryo forms a radially symmetric cup-shaped epithelium, the Epiblast (Epi) containing around 300 cells that is attached to the Extra embryonic Ectoderm (ExE) on the proximal side and embedded in another epithelium, the Visceral Endoderm (VE) a derivative of the PrE. At this time, a symmetry-breaking event defines a group of cells within the VE that move to one side and give rise to the Anterior Visceral Endoderm (AVE), a cellular collective that defines the anterior region of the embryo on the adjacent Epi cells and, as a result, establishes the anteroposterior (AP) axis of the embryo. At this stage, the Epi experiences widespread BMP, Nodal and Wnt signalling but the AVE, acting as a source of antagonists of these signalling pathways (Cerl, Lefty1 and Dkk1), restricts their effects to the side of the epithelium opposite to the AVE where they induce the localised expression of T/Bra, the start of gastrulation by initiation of the Primitive Streak and axial extension at the posterior end. Figure part adapted from [8].

approaches: identification of mutations that disrupt the process of the genes affected in the mutations and, guided by the molecular nature of the protein encoded by those genes, the assignation of molecular events to the process $[9,10]$; this approach has been successful. However, while it allows some insights into the processes at work, it has limitations. It reveals what is necessary but not what is sufficient, it can conflate correlation and causation, has problems with redundancies and cannot easily probe for the role of mechanical forces, which experiments suggest also play a role in the early events $[11,12]$. A complementary approach requires an experimental system that allows a precise and reliable perturbation of the patterning process, the quantitative analysis of the experimental results and, if possible, the exploitation of available genetics. Importantly, such system should mimic the embryo.

We have established a non adherent culture system for mouse Embryonic Stem Cells (ESCs) in which small aggregates of cells undergo symmetry-breaking, polarisation of gene expression and axial development in a reproducible manner that mirrors events in embryos [13-15]. These polarised aggregates, that we call Gastruloids, provide a versatile and useful system that, in combination with genetics, fulfils many the requirements for experimental manipulations to study pattern formation in cell ensembles.

Here we use the Gastruloid system to study the signalling events that lead to their early patterning: the symmetry-breaking event and the polarised expression of Brachyury ( $\mathrm{T} / \mathrm{Bra}$ ) that, in the embryo, reflects the establishment of the AP axis and presages the process of gastrulation. We find that during 24-48h of culture, interactions between Nodal and Wnt signalling promote an intrinsic symmetry-breaking event that is recorded in the expression of $T / B r a$; BMP signalling is not required for these events. These results confirm that Gastruloids behave like cells in the epiblast [13] and lead us to suggest that a similar spontaneous symmetry-breaking event occurs in the embryo where biases from the extraembryonic tissues, ensure its reproducible location next to the Extraembryonic Ectoderm. We also show that Wnt signalling plays an important role in the amplification and stabilisation of these events.

Our results establish Gastruloids as a robust experimental system to analyse mechanisms of fate decisions and pattern formation in mammals and provide some insights about the interactions between signalling pathways in the patterning of embryos, in particular about the role of epithelia and the extraembryonic tissues.

\section{Materials and Methods}

Cell lines and routine cell culture: Bra::GFP [16], Nodal ${ }^{\text {condH } B E:: Y F P}$ [17], AR8::mCherry (Smad2/3 reporter) [18], IBRE4-TA-Cerulean (BMP reporter) [18], Spry4::H2B-Venus (see below) and TCF/LEF::mCherry (TLC2) [19, 20] were cultured in serum supplemented with LIF and fotal bovine serum (ESL medium) on gelatinised tissue-culture flasks and passaged every second day as previously described $[14,19,21-23]$. If cells were not being passaged, half 
bioRxiv preprint doi: https://doi.org/10.1101/051722; this version posted May 13, 2016. The copyright holder for this preprint (which was not certified by peer review) is the author/funder, who has granted bioRxiv a license to display the preprint in perpetuity. It is made available under aCC-BY 4.0 International license.

the medium in the tissue culture flask was replaced with ESL. All cell lines were routinely tested and confirmed free from mycoplasma.

Generation of Cell Lines The Spry4::H2B-Venus reporter cell line was generated by combining knockout first targeting arms of the EUCOMM project [24] with a H2B-Venus reporter cassette and a neomycin resistance gene driven from a human $\beta$-actin promoter. This construct was integrated by homologous recombination into a cell line carrying a doxycycline-inducible Gata4-mCherry construct described in [25]. This cell contains a constitutively active Cerulean fluorescent protein to aid in image analysis [25]. See supplemental materials and methods for a detailed description of the generation of this cell line.

Immunofluorescence and Microscopy: Gastruloids from the Nodal condHBE::YFP [17] cell line were fixed and stained for YFP, Brachyury and Nanog according to the protocol previously described [15]. Hoechst3342 was used to mark the nuclei (see Table $\mathbf{S 1}$ for the antibodies used and their dilutions). Confocal z-stacks of Gastruloids were generated using an LSM700 (Zeiss) on a Zeiss Axiovert $200 \mathrm{M}$ using a 40 EC Plan-NeoFluar 1.3 NA DIC oil-immersion objective. Hoechst3342, Alexa-488, 568 and 633 were sequentially excited with 405, 488, 555 and $639 \mathrm{~nm}$ diode lasers respectively as previously described [23]. Data capture was carried out using Zen2010 v6 (Carl Zeiss Microscopy Ltd, Cambridge UK) and analysis of the average pixel intensities within each nucleus within a given z-plane was performed in Fiji [26]. The z-stacks were acquired of at least 4 Gastruloids per condition with a $\mathrm{z}$-interval of $2.11 \mu \mathrm{m}$ for a maximum of $42.2 \mu \mathrm{m}$.

Widefield, single-time point images of Gastruloids were acquired using a Zeiss AxioObserver.Z1 (Carl Zeiss, UK) in a humidified $\mathrm{CO}_{2}$ incubator $\left(5 \% \mathrm{CO}_{2}\right.$, $37^{\circ} \mathrm{C}$ ) with a $20 \mathrm{x}$ LD Plan-Neofluar 0.4 NA Ph2 objective with the correction collar set to image through plastic. Illumination was provided by an LED whitelight system (Laser2000, Kettering, UK) in combination with filter cubes GFP-1828A-ZHE (Semrock, NY, USA), YFP-2427B-ZHE (Semrock, NY, USA) and Filter Set 45 (Carl Zeiss Microscopy Ltd. Cambridge, UK) used for GFP, YFP and RFP respectively and emitted light recorded using a back-illuminated iXon888 Ultra EMCCD (Andor, UK). Images were analysed using the ImageJ image processing package Fiji [26] and plugins therein as previously described [15]. Briefly, the florescence intensity was measured by a line of interest (LOI) drawn from the posterior to anterior region of the Gastruloid with the LOI width set to half the diameter of the Gastruloid at 48h (100px with the $20 \mathrm{x}$ objective). The background for each position was measured and subtracted from the fluorescence for each Gastruloid. Shape-descriptors were generated by converting brightfield images of Gastruloids to binary images and measuring them by particle detection.
Fluorescence levels were normalised to the maximum obtained in following Chi stimulation, and the maximum length of each Gastruloid was rescaled 1 unit. Average fluorescence traces of Gastruloids \pm S.D. are shown.

For live imaging experiments, each well of a 96-well plate containing individual Gastruloids was imaged as described above using both the 20x (24-72h) and the 10x (72-96h) objectives described above, and images captured every $30 \mathrm{~min}$ for a maximum of $96 \mathrm{~h}$. All images were analysed in Fiji [26] using the LOI interpolator [27] with the LOI set to a width of 100px.

\section{Gastruloid culture and application of specific} signals: Aggregates of mouse ESCs were generated as previously described $[13,15]$. Briefly, mouse ESCs suspended in $40 \mu \mathrm{l}$ droplets of N2B27 were plated in round-bottomed low-adhesion 96-well plates and left undisturbed for $48 \mathrm{~h}$, following which, they were competent to respond to specific signals. The number of cells within each droplet was optimised to ensure that the size of the Gastruloid was $\sim 150 \mu \mathrm{m}$ in diameter at $48 \mathrm{~h}$; this generally consisted of 400 cells $/ 40 \mu \mathrm{l} /$ well (See Table S2 for the numbers of cells required for each cell line used in this study). In experiments which required the addition of specific factors to Gastruloids on the second day of aggregation (24-48h), $20 \mu \mathrm{l}$ medium was carefully removed with a multichannel pipette, and $20 \mu \mathrm{l}$ of N2B27 containing twice the concentration of factors was added. This method was preferable to the addition of smaller volumes containing higher concentrations of agonist/antagonists, as the data from these experiments showed more variation between Gastruloids (DAT, PB-J, AMA unpublished). Control experiments (e.g. DMSO or N2B27 only) showed that replacement of half the medium at this stage did not significantly alter the ability of Gastruloids to respond to signals on the third day. The next day, $150 \mu$ l fresh N2B27 was added to each of the wells with a multichannel pipette for $1 \mathrm{~h}$ to wash the Gastruloids; a time delay ensured that sample loss was prevented. Following washing, $150 \mu \mathrm{l}$ N2B27 containing the required factors was then applied. The small molecules used in this study and their concentrations are described in Table S3.

Quantitative RT-PCR Gastruloids ( $n=64$ per time-point) from T/Bra::GFP mouse ESCs, subjected to a Chi or DMSO pulse (between 48 and $72 \mathrm{~h} \mathrm{AA}$ ), harvested at 48 or $72 \mathrm{~h} \mathrm{AA}$, trypsinised, pelleted and RNA extracted using the RNeasy Mini kit (Qiagen, 74104) according to the manufacturers instruction as previously described [22]. Samples were normalised to the housekeeping gene PPIA. The primers for BMP4, Cerl, Chordin, DKK, FGF4, FGF5, FGF8, Lefty1, Nodal, Ppia, Spry4, Wnt3 and Wnt3a are described in Table S4.

Orientation of Gastruloids To define the AP orientation of Gastruloids, we have assigned the point of 
bioRxiv preprint doi: https://doi.org/10.1101/051722; this version posted May 13, 2016. The copyright holder for this preprint (which was not certified by peer review) is the author/funder, who has granted bioRxiv a license to display the preprint in perpetuity. It is made available under aCC-BY 4.0 International license.

A
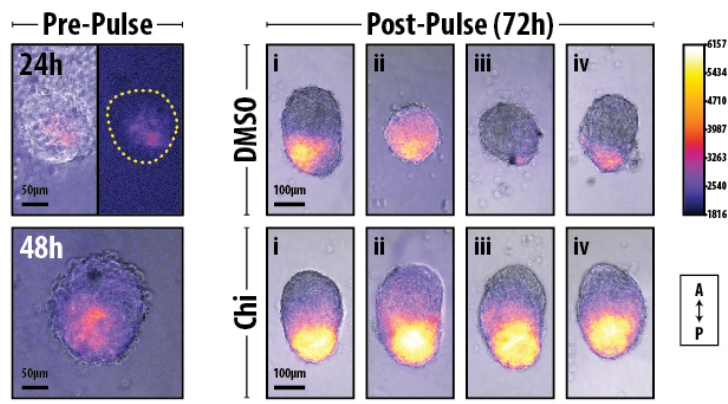

B

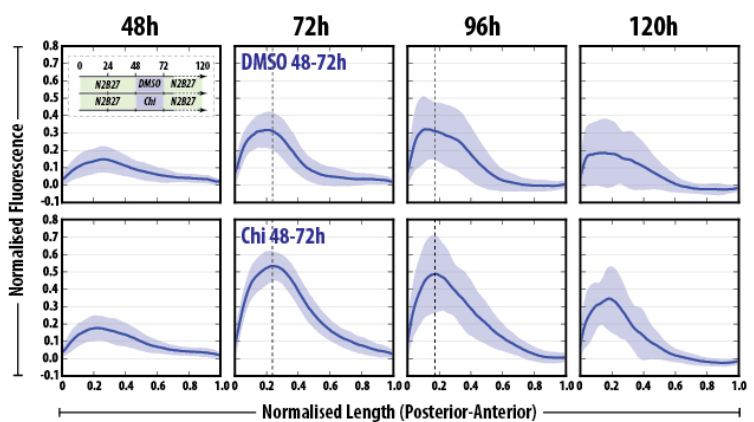

D
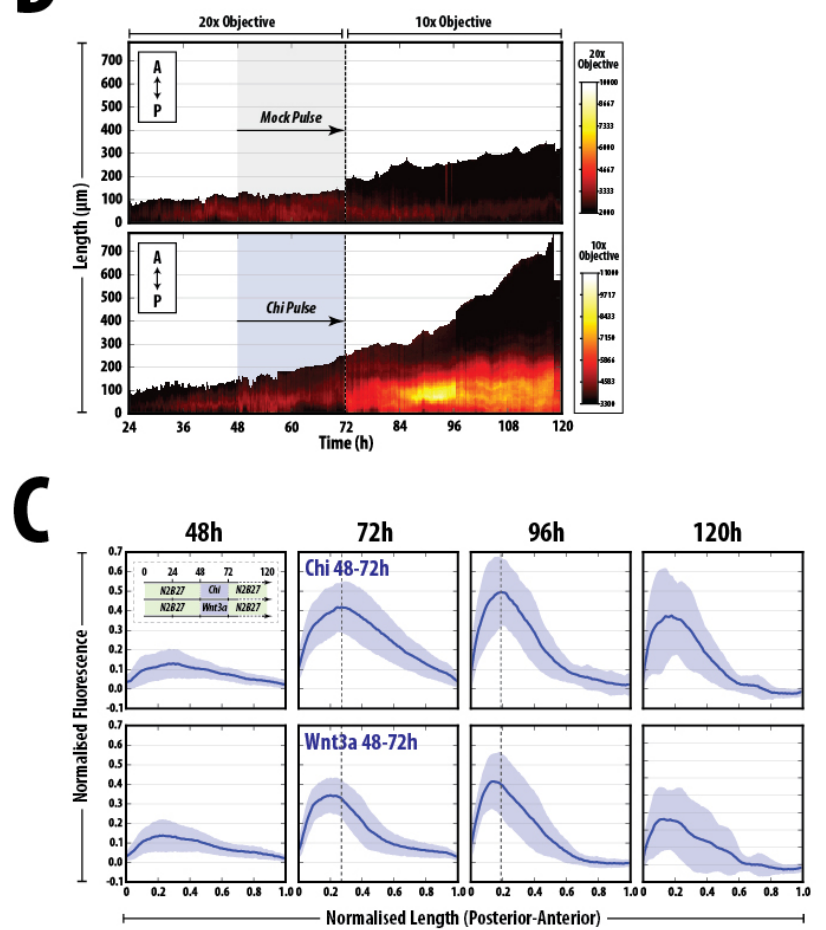

Figure 2: Symmetry-breaking and polarisation in the absence of signalling. Gastruloids generated from T/Bra::GFP were formed and maintained in N2B27 medium for the duration of the experiment and subjected to a $24 \mathrm{~h}$ pulse of Chi or DMSO between 48 and $72 \mathrm{~h}$ after aggregation (AA). (A) Morphology and expression of T/Bra::GFP at 24 and 48h prior to the Chi pulse (left), and examples (i-iv) of Gastruloids following either the Chi or DMSO pulse (right). Chi stimulation increases the robustness of the response and reproducibility of the phenotype. Anteroposterior orientation indicated (bottom right). (B) Quantification of T/Bra::GFP reporter expression in individual Gastruloids over time from one replicate experiment. The maximum length of each Gastruloid is rescaled to 1 unit and the fluorescence is normalised to the maximum fluorescence from the Chi condition. (C) Quantification of T/Bra::GFP Gastruloids following stimulation with either Chi or Wnt3a. (D) Live imaging of one representative Gastruloid subjected to a pulse of DMSO (top) or Chir (bottom) between 48 and $72 \mathrm{~h} \mathrm{AA}$. Shown is the length of the Gastruloid over time (posterior $=0 \mu \mathrm{m}$ ) and the fluorescence intensity of the reporter (colour). Early time-points (24-72h AA) imaged with a higher power objective. The schematic of the experimental design for Gastruloid stimulation is indicated as inserts in (B) and (C). Horizontal lines at 72 and $96 \mathrm{~h} \mathrm{AA}$ in (B) and (C) indicate the maximum fluorescence of the Chi pulse condition

T/Bra::GFP expression as the 'Posterior', as the primitive streak, which forms in the posterior of embryo, is the site of $T / B r a$ expression in the embryo [28-30].

\section{Results}

\subsection{Intrinsic Patterning in the Gastru- loids}

As a reference for the evaluation and interpretation of our results, a summary of the main events that lead to the establishment of the anteroposterior (AP) axis in the mouse embryo is provided in Fig. 1. This process confines the start of gastrulation to a cell population in the proximal posterior edge of the embryo, next to the Extraembryonic Ectoderm, through a sequence of carefully choreographed interactions between extraembryonic and embryonic tissues mediated by Nodal, BMP and Wnt signalling. The relationship between these three signals has been established in genetic experi- ments and places Nodal and Wnt signalling in the epiblast as the effective target of the process [31, 32].

To investigate initial patterning mechanisms during development, and to circumvent the difficulties involved in experimentation with embryos at this stage of development, we utilised our non-adherent culture method [13-15]. We have reported before that in this system, small numbers of ESCs upon specific culture treatment will undergo symmetry-breaking and axial elongation; here we confirm this. Using a T/Bra::GFP line that identifies the Bra-expressing cells as the posterior of the Gastruloids, we set out to study the mechanisms involved in the patterning of Gastruloids. (See Materials and Methods). After the initial aggregation period, Gastruloid cultures were kept individually for 2 days in N2B27 (Fig. 2A). If they were maintained in N2B27 for a further $72 \mathrm{~h}$ (120h AA), a variety of patterns were observed with a large degree of interexperimental variation. In general about $50 \%$ of individual Gastruloids exhibited different degrees of axial 
bioRxiv preprint doi: https://doi.org/10.1101/051722; this version posted May 13,2016. The copyright holder for this preprint (which was
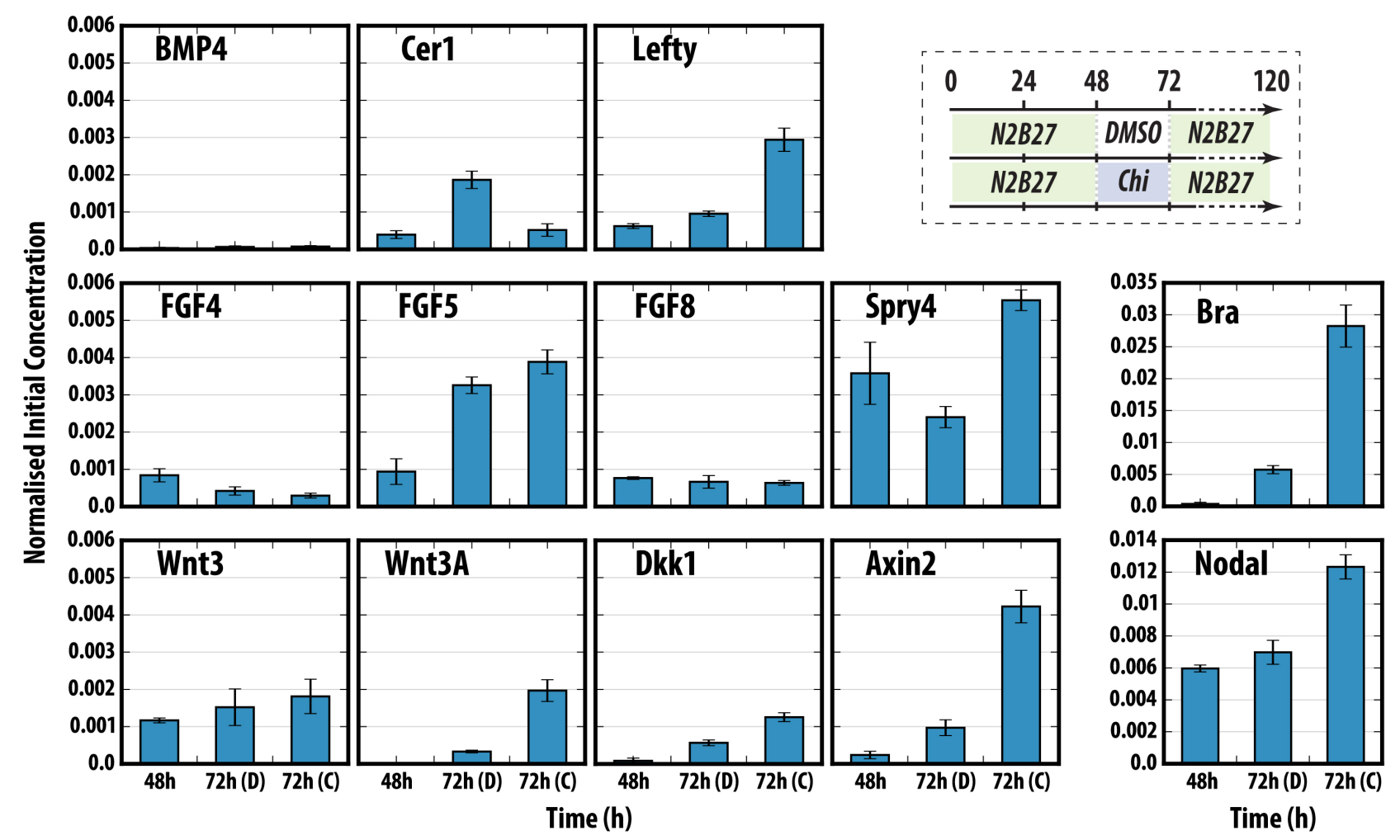

Figure 3: Quantitative PCR analysis of specific genes in early Gastruloids development, following a Chi pulse. Gastruloids generated from T/Bra::GFP mouse ESCs were harvested at 48 and $72 \mathrm{~h}$ with $(72 \mathrm{~h}(\mathrm{C})$ ) or without (72h (D)) a Chir pulse prior to RNA extraction and qRT-PCR analysis for the indicated genes. Data normalised to the housekeeping gene Ppia and is representative of 2 replicate experiments; error bars represent standard deviation of triplicate samples. Schematic of experimental design shown top right.

elongation and T/Bra::GFP polarisation while the rest were very varied. Following stimulation by signals such as the Wnt/ $\beta$-Catenin agonist (Chi) between $48-72 \mathrm{~h}$ AA, over $85 \%$ of the Gastruloids underwent axial elongation. Our results suggest that there is an intrinsic symmetry-breaking event between 24 and $48 \mathrm{~h} \mathrm{AA}$ and that exposure to Wnt/ $\beta$-Catenin signalling plays a role in its stabilisation.

Due to the reproducibility of these structures in culture and the ease with which they can be manipulated by the addition of specific signalling molecules, we were able to utilise this technique to assess how the initial patterning event in the Gastruloids is established.

Analysis of Gastruloids 24h AA revealed weak, spotty expression of $\mathrm{T} / \mathrm{Bra}: \mathrm{GFP}$ with a proportion already displaying signs of biased expression towards one pole (Fig. 2). By 48h AA in N2B27, the expression levels of $\mathrm{T} / \mathrm{Bra}: \mathrm{GFP}$ had risen and a more prominent polarisation and regionalisation of the reporter was observed in several Gastruloids (Fig. 2A). T/Bra::GFP was observed in one hemisphere of the Gastruloids, which tended to show a slight distortion in their shape from spherical to slightly ovoid as previously reported [13]. Addition of Chi at $48 \mathrm{~h}$ increased both the levels of T/Bra::GFP and polarity by $72 \mathrm{~h}$ AA (Fig. 2). If Gastruloids were treated with the vehicle control (DMSO), regionalised expression of the reporters could also observed, however the fluorescence levels were generally lower, with a higher degree of vari- ation both within and between experimental replicates. This suggests that increased Wnt/ $\beta$-Catenin signalling modulates the reproducibility and stability of the established pattern (Table S5).

To garner a better understanding of the heterogeneities in levels of T/Bra::GFP expression between the Gastruloids over time, we quantified the fluorescence in a posterior to anterior direction along the spine of the Gastrulioids (Fig. 2B,C; see Materials and Methods and [15]). T/Bra::GFP expression increased transiently over time, peaking at 96h AA (Fig. 2B,C), and was localised predominantly towards the posterior region (the origin) at all time-points (polarisation). Chi stimulation results in a lower standard deviation and a higher maximum fluorescence than the DMSO control and the expression of the reporter is maintained at the later time-points following Chi treatment (Fig. 2B). Stimulation Wnt3a resulted in a similar fluorescence trace compared with Chi (Fig. 2C), however the heterogeneity between individual Gastruloids is reduced and the higher expression is confined to a narrower region of the Gastruloid (increased polarity; Fig. 2C).

Next, to assess the evolution of T/Bra::GFP expression over time, we used live-cell microscopy to image the Gastruloids from 24 to $120 \mathrm{~h} \mathrm{AA}$ following a pulse of Chi or vehicle (Fig. 2D). Prior to stimulation, the size of the Gastruloids increased steadily over time with a concomitant increase in the levels of T/Bra::GFP to- 
bioRxiv preprint doi: https://doi.org/10.1101/051722; this version posted May 13, 2016. The copyright holder for this preprint (which was not certified by peer review) is the author/funder, who has granted bioRxiv a license to display the preprint in perpetuity. It is made available under aCC-BY 4.0 International license.

A

\begin{tabular}{ccccc}
0 & 24 & 48 & 72 & 120 \\
\hline$N 2 B 27$ & DMSO & $N 2 B 27$ \\
\hline$N 2 B 27$ & Chi & $N 2 B 27$ \\
\hline
\end{tabular}

C

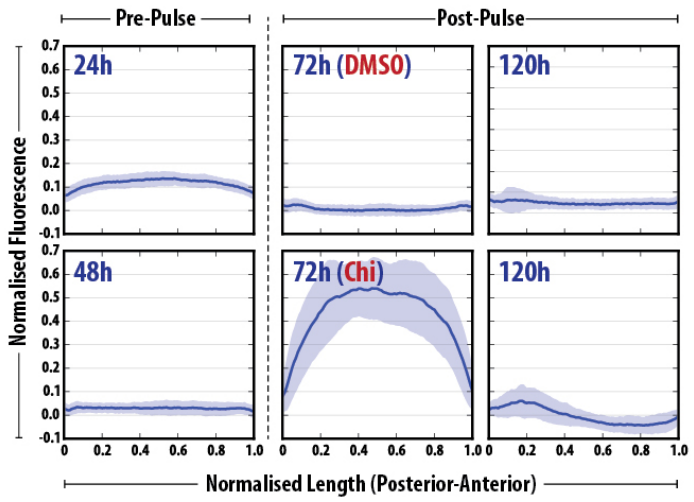

B
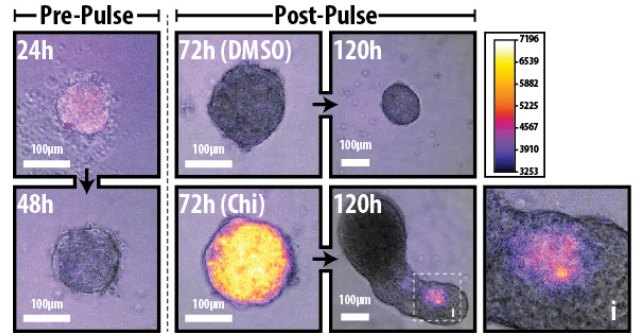

$\mathbf{B}^{\prime}$

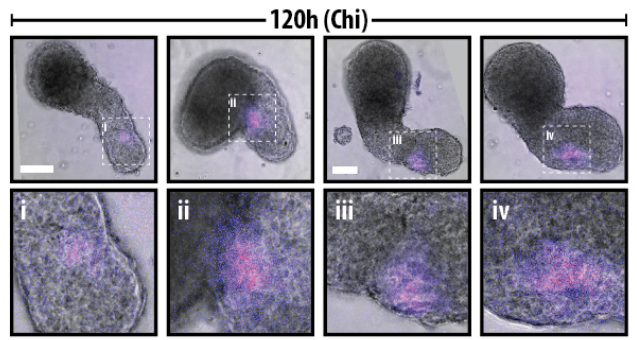

D
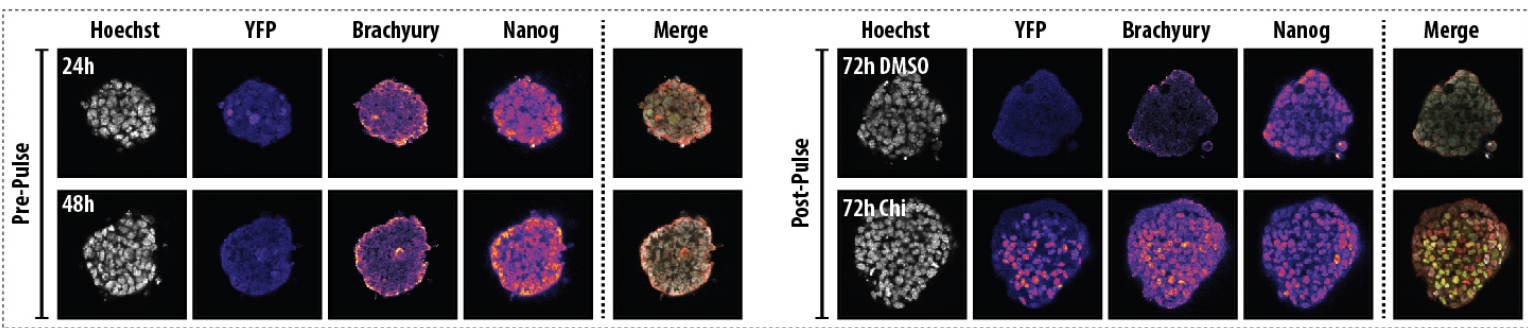

Figure 4: Expression of Nodal during early aggregate formation. (A) Schematic of the experimental design for Gastruloid stimulation. (B) Gastruloids formed from Nodal ${ }^{c o n d H B E:: Y F P}$ (Nodal reporter) ESCs either maintained in N2B27 or stimulated with a pulse of Chi or vehicle between 48 and $72 \mathrm{~h} \mathrm{AA}$ were imaged by wide-field fluorescence microscopy at 24, 48 and 72h AA. (B') Gastruloids at 120h AA demonstrating a single region of Nodal expression slightly towards one side of the elongate; magnified regions (i-iv) indicated by hashed white box. (C) Quantitative analysis of the Gastruloids at the aforementioned time-points with their maximum length set to 1 unit and normalising their fluorescence to the maximum fluorescence of the Chi pulse at $72 \mathrm{~h}$ AA. (D) A single slice through a confocal stack of the Nodal reporter Gastruloids stained for YFP, T/Bra and Nanog. See (Fig. S2) for the quantification of the confocal images. Notice the increase in Nodal expression after Chi stimulation.

wards one pole, the expression of which became more apparent at approximately 36h AA (Fig. 2D). The addition of Chi at $48 \mathrm{~h}$ did not have an immediate effect on the expression of the reporter until 72h AA, when a flash of T/Bra::GFP expression was observed throughout the aggregate; higher expression occurred towards the region that was already expressing T/Bra::GFP (Fig. 2D). Between 72-84h AA, T/Bra::GFP was down-regulated in the region that was previously not expressing the reporter and was up-regulated in the region that was already expressing T/Bra::GFP. This suggests that once a T/Bra::GFP domain is specified, only that region can stably respond.

Altogether, these data suggest that by $48 \mathrm{~h} \mathrm{AA}$, the Gastruloids have developed an initial patterning, intrinsically driven, prior to the addition of signalling factors. Furthermore, increased Wnt $/ \beta$-Catenin signalling by the addition of Chi increases the reproducibility both intra- and inter-experimentally, and ro- bustness of the final phenotype and the patterning.

\subsection{Gene Expression Analysis of Early Stage Gastruloids}

The observation that the patterning of Gastruloids occurs in the absence of an external input led us to search for the expression of elements of the signalling pathways known to participate in symmetry-breaking in the embryo (Fig. 1). At 48h AA, Gastruloids express low levels of FGF4, 5, Axin2, Wnt3, Nodal and Lefty1 (Fig. 3), all of which are expressed in the epiblast of the E6.0 embryo. Additionally, we detected no expression of $B M P 4, D k k$ or Chordin with very low expression of Cerberus (Fig. 3). Noggin, an antagonist of BMP signalling, is not expressed in the early stages of development and its absence of expression serves as a baseline for the others (data not shown). This pattern is consolidated in the absence of any external input with 

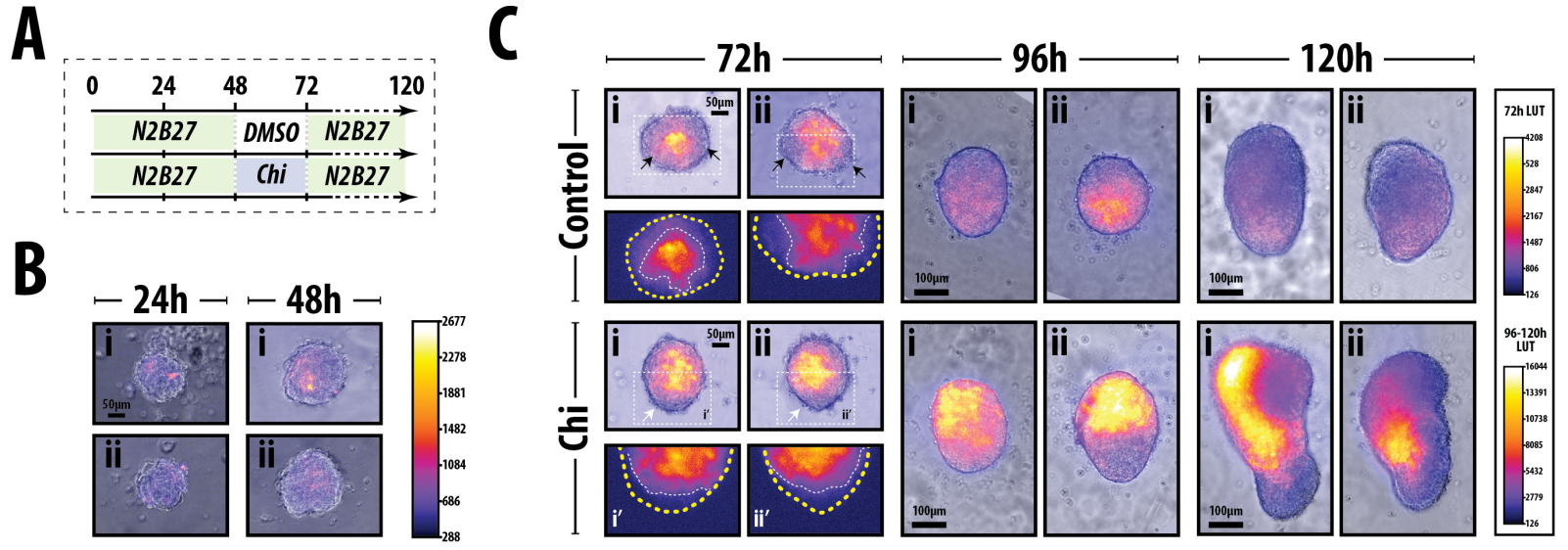

Figure 5: Expression of AR8::mCherry following Wnt/ $\beta$-Catenin activation. (A) Schematic of the experimental conditions. (B) Expression of the AR8::mCherry reporter after 24 and 48h. (C) Expression of the reporter in two representative Gastruloids following either DMSO or Chi at 72, 96 and 120h. Magnified regions (hashed white line) at $72 \mathrm{~h}$ are shown, indicating regions of the gastruloid that do not express the reproter. The $24 \mathrm{~h}$ time-point in (B) was taken from a seperate experiment with identical imaging conditions as the $48 \mathrm{~h}$ condition and all time-points in (C). The lookup tables for 24/48h, $72 \mathrm{~h}$ and $96 / 120 \mathrm{~h}$ has been rescaled to aid in the visualisation and localisation of the reporter when expressed at low levels.

increases in expression of Nodal, Lefty1 and FGF5, decreases in $F G F 4$ expression and the emergence, at low levels, of $W n t 3 a$ at $72 \mathrm{~h} \mathrm{AA}$ (Fig. 3; 72h(D)). Exposure to Chi during 48 and $72 \mathrm{~h}$ AA leads to an increase in Axin2, Nodal, Lefty, Wnt3a and Dkk, all targets of Wnt signalling (Fig. 3; $72 \mathrm{~h}(\mathrm{C})$ ).

These results confirm that Gastruloids resemble the cells in the epiblast and also provide gene expression landmarks to correlate their development with events in embryos e.g. FGF5 is a marker of the E5-E6.5 epiblast and $W n t 3 a$ and $D k k$ in the embryo, characterise the onset of gastrulation. Altogether these observations lead us to suggest that Gastruloids at 48h AA correspond, approximately, to $\mathrm{E} 6.0( \pm 0.5)$ in the embryo and $72 \mathrm{~h}$ AA to E7.0 $( \pm 0.5)$. Further observations below confirm and extend this correspondence (Fig. 3).

\subsection{Early Nodal expression and sig- nalling are associated with the sym- metry breaking event}

The characterisation of the patterning and gene expression of early Gastruloids suggests that the Nodal/TGF $\beta$ signalling pathway may be involved in the initial patterning event. To address this further, we assessed the pattern of expression of two reporters for Nodal activity in the Gastruloids from the early stages of aggregation (24h) up to $120 \mathrm{~h} \mathrm{AA}$. Gastruloids were generated from ESCs containing a Nodal ${ }^{\operatorname{cond} H B E:: Y F P}$ transcriptional reporter [17] (hereon referred to as the Nodal Reporter; (Fig. 4) or a Smad2/3 Nodal signalling reporter (AR8::mCherry) [18] (hereon referred to as the Signalling Reporter Fig. 5).

At $24 \mathrm{~h}$ AA, most of the cells within each Gastruloid expressed low levels of the Nodal reporter heterogeneously, with no evidence of a bias to any particular region and by $48 \mathrm{~h}$ AA it was down-regulated throughout each Gastruloid (Fig. 4A-C). Gastruloids formed from the signalling reporter showed expression prior to the addition of Chi (Fig. 5) however, by contrast to the Nodal reporter, the signalling reporter was expressed in discrete punctate patterns that varied from Gastruloid to Gastruloid that increased, in number and intensity, between 24 and 48h AA.

Whereas continued culture in N2B27 maintained low expression of the Nodal reporter (Fig. 4A,B), following a Chi pulse between 48 and $72 \mathrm{~h} \mathrm{AA}$, a dramatic increase in fluorescence throughout the whole Gastruloid was observed (Fig. 4A,B), that correlated with the expression within the epiblast at E4.5 and 5.5 [17]. Similarly, the signalling reporter was up-regulated following addition of Chi (Fig. 5C), however, at the end of the Chi pulse (72h AA), we observed in many Gastruloids a crescent free of the expression of the signalling reporter at the future posterior end (Fig. 5C). This pattern was also observed in the DMSO treated Gastruloids control, but regions of non-expression were more likely to appear in multiple locations (Fig. 5C). As with the Nodal reporter, vehicle treatment failed to up-regulate the signalling reporter to the same extent as Chi treated Gastruloids (Fig. 5C), indicating that Wnt/ $\beta$-Catenin signalling was necessary for the enhancement and maintenance of Nodal signalling within one, polar region of the Gastruloids.

Between 96 and 120h AA, Nodal reporter Gastruloids treated with Chi confined Nodal expression to the elongating region and gradually reduced their expression (Fig. 4B,B'). By 120h AA these Gastruloids developed a polarised elongation and we note the emergence of high reporter activity in a group of cells centred at the anterior edge of the elongate; these cells may correspond to an attempt to establish a node as they mimic Nodal expression in the embryo [33, 34]. 
A

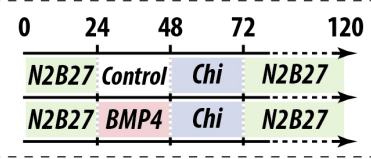

B

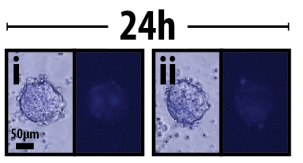

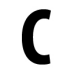
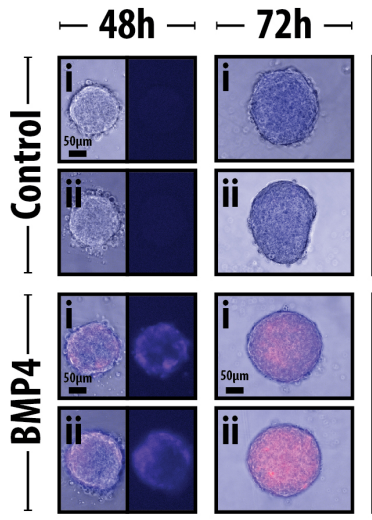
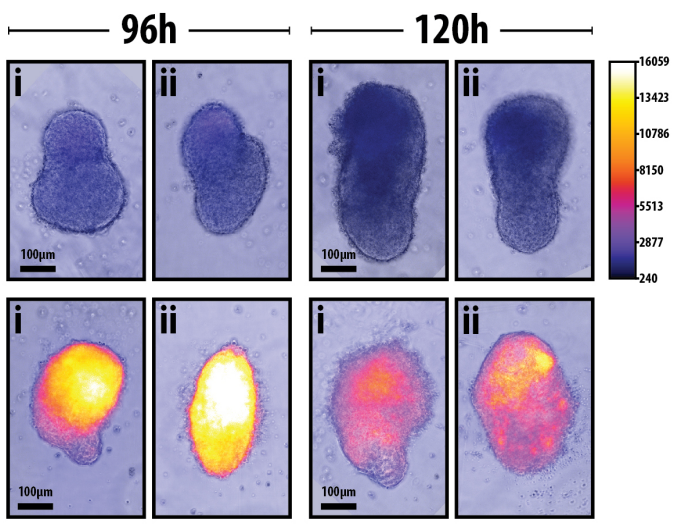

Figure 6: Expression of IBRE4-TA-Cerulean in Chi pulsed Gastruloids either pre-treated with vehicle or BMP4 for 24h. (A) Schematic of treatment and conditions. Cont; Control ( $\mathrm{HCl}+0.1 \% \mathrm{BSA})$. Gastruloids imaged at 24h (B) and 47-120h (C) in the conditions described in (A). By 24h, there is very low BMP signalling. Following BMP pretreatment greatly increases the fluorescence of the reporter. Scalebar indicates $100 \mu \mathrm{m}$, lookup table displayed in $(\mathrm{C})$ corresponds all images in this figure.

In support of this, in some instances, we observe low levels of the reporter restricted to one side of the aggregate, much as is the case for Nodal in the embryo (Fig. 4B'i-iv).

Finally, we sought to directly probe for the expression of $\mathrm{T} / \mathrm{Bra}$ and Nanog within Nodal reporter Gastruloids to assess a) the correlation between Nodal expression and the acquisition of a posterior fate and b) to understand the timing of the initial symmetrybreaking event with respect to the loss of pluripotency (Nanog) and initiation of differentiation (T/Bra). Nodal reporter Gastruloids were fixed and stained for GFP, Brachyury and Nanog, and their expression assessed by confocal microscopy (Fig. 4C).

By $24 \mathrm{~h}$ AA, Nodal was expressed at low levels in a heterogeneous manner, similar to that seen by widefield microscopy (Fig. 4D, Fig. S1). Nanog was also expressed in a heterogeneous manner, with a broad distribution in fluorescence values (Fig. 4D, Fig. S1); there was no detectable above-baseline expression of Brachyury at this stage.

In Gastruloids maintained in N2B27 from 48h to $72 \mathrm{~h}$, Nodal expression remained low with no increase in the expression of Brachyury and a gradual reduction in the expression of Nanog (Fig. 4D, Fig. S1). This was reflected in the increasing correlation between Nanog, Brachyury and Nanog as their expression gradually reduced to background (Table S6). Stimulation with Chi from 48-72h increased the fluorescence of Nodal, Brachyury and Nanog in a heterogeneous manner, suggesting a commitment to a differentiated state (Fig. 4D, Fig. S1); the heterogeneous expression of Nodal in this region bears similarity to that in the embryo at E6.5[17]. Furthermore, there is evidence for expression of Nanog in this region at this time.

This suggests that events in the Gastruloids mimic events in embryos and that the period around $48-72 \mathrm{~h}$ is related to the onset of gastrulation, and we would suggest here that it might encompass the E6.5-E7.5 in the embryo. In these conditions, the expression of Nodal and Brachyury was strongly correlated (Table S6).

\subsection{BMP4 signalling is undetectable during Gastruloid Progression}

In the embryo, genetic experiments have demonstrated the need for BMP signalling from the Extra embryonic regions for the expression and localisation of Nodal and Wnt signalling to the proximal posterior region of the embryo, however our data suggests that there is no extraembryonic component in the Gastruloids. To test this further, we investigated the activity of BMP signalling using a Smad1,5,7 reporter (IBRE4TA-Cerulean; hereon known as the BMP reporter) [18] and measured its expression within Gastruloids treated either with DMSO or with Chi (48-72h AA) that had either been pre-treated with BMP 4 or vehicle (Fig. 6).

In Gastruloids only treated with a Chi pulse (control) we did not detect any significant increase in the expression of the BMP reporter from 24-120h AA. To test whether the cells are responsive to BMP at this stage, Gastruloids were pre-treated with BMP4 prior to the Chi pulse. BMP4 Pre-treatment greatly enhanced the activity of the reporter, with the majority of the expression located in the 'anterior' region (Fig. 6C).

Although it is possible that the reporter may not be sensitive enough to detect very low levels of signalling, these results demonstrate the lack of signalling in the Gastruloids and further support the notion that Gastruloids are made up of epiblast cells. In addition it also suggest that BMP signalling is not needed for the symmetry-breaking event itself nor the expression of $\mathrm{T} /$ Bra (see next section). Whatever low level of BMP signalling exists within the Gastruloids, it is not modified by Wnt signalling. 
bioRxiv preprint doi: https://doi.org/10.1101/051722; this version posted May 13, 2016. The copyright holder for this preprint (which was not certified by peer review) is the author/funder, who has granted bioRxiv a license to display the preprint in perpetuity. It is made available under aCC-BY 4.0 International license.

A

\begin{tabular}{|c|c|c|c|c|}
\hline N2B27 DMSO & Chi & N2B27 & BMP4 & DMH1 \\
\hline N2B27, (i) & Chi & N2B27 & Nodal & SB43 \\
\hline N2B27 (ii) & Chi & N2B27 & & \\
\hline
\end{tabular}

B
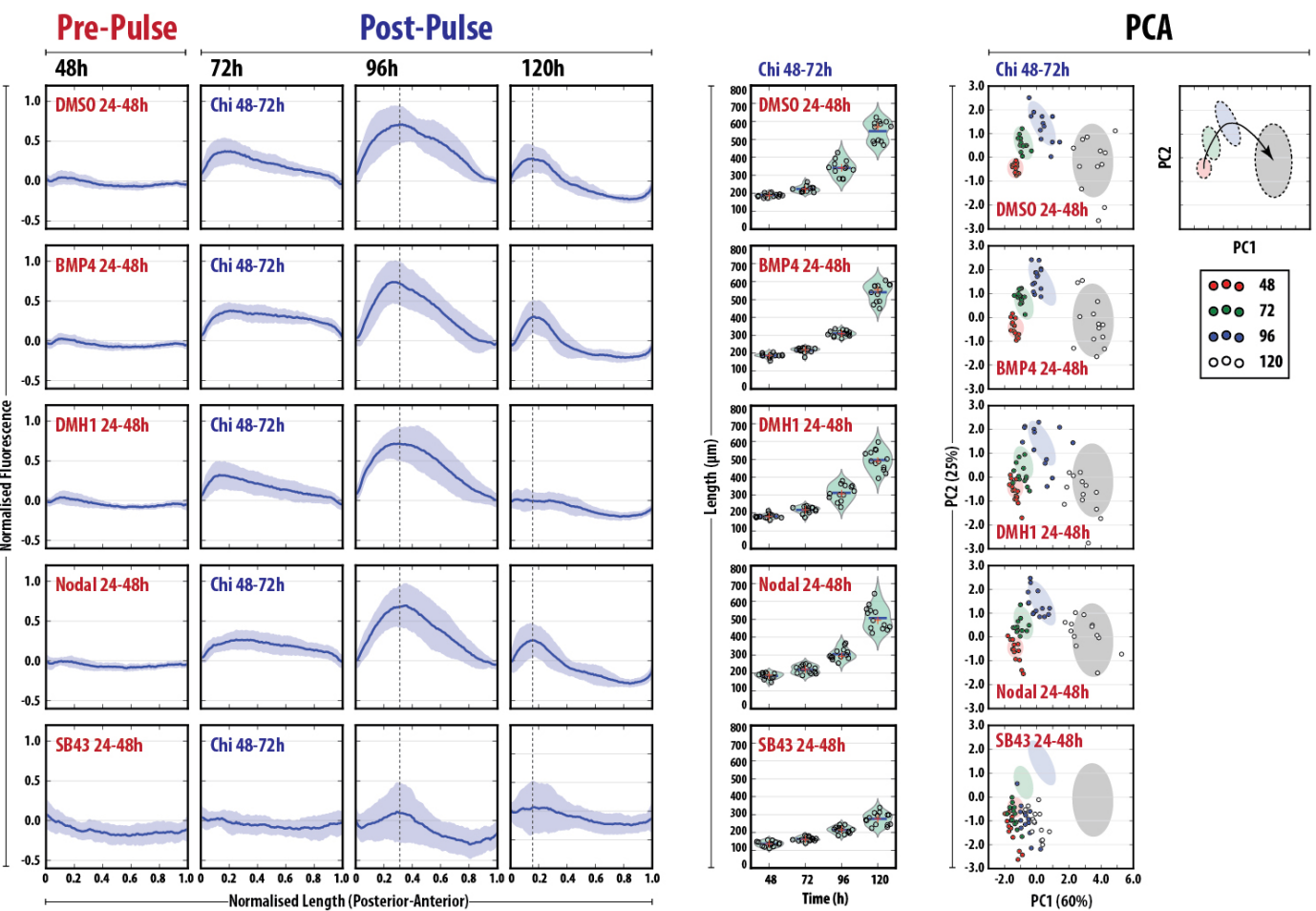

Figure 7: Quantitative analysis of T/Bra::GFP Gastruloids reveals importance of early Nodal signalling for correct patterning. (A) A schematic of the experimental design for Gastruloid stimulation. Gastruloids, aggregated in N2B27 (green shading) were treated with either vehicle, activators (i) or inhibitors (ii) of the Nodal and BMP signalling pathways shown in the table on the right, for $24 \mathrm{~h}$. Following wash-off of these factors, all Gastruloids were treated with a $24 \mathrm{~h}$ pulse of Chi followed by sustained culture in N2B27. (B) Individual Gastruloids treated with vehicle, BMP4, DMH1, Nodal or SB43 were imaged by wide-field fluorescence microscopy and quantitatively analysed for the fluorescence (left panel) and length (middle panel). Principal Component Analysis (right) was used to reduce the dimensions of the data, revealing distinct clusters which corresponded to the specific time-points. The indicated percentage for the explained variance is denoted in the axis labels for PC1 and PC2. The fluorescence plots of the left of (B) were normalised to the maximum fluorescence of the control, and the maximum length of each Gastruloid was rescaled to 1 unit. Horizontal dashed lines at 72 and 96h AA in B indicate the maximum fluorescence of the Chi pulse condition.

\subsection{Functional analysis of signalling in the early patterning of Gastruloids}

Our data suggest that interactions between the TGF $\beta$, FGF and Wnt/ $\beta$-Catenin signalling before the pulse of Chi and as the Gastruloids polarise, mediate the symmetry-breaking event. To test this, we used agonists and antagonists of these pathways in the form of small molecules and recombinant proteins that target Nodal, BMP, FGF and Wnt/ $\beta$-Catenin signalling on the day before the Chi pulse (Figs. $\mathbf{7 A}, \mathbf{8 A}, \mathbf{9 A}$ ). It is to be noted that gentle mechanical and volumetric disruptions on the second day (24-48h AA) did not unduly affect the patterning and development of the Gastruloids (see Materials and Methods). As a read-out of the patterning events, we used T/Bra::GFP Gastruloids and recorded the fluorescence and morphology by widefield microscopy (Figs. 7B, 8B, 9B).

As well as plotting the normalised fluorescence and the length of the Gastruloids for each specific treatment (left and middle panels of Figs. 2A-C, 7B, 8B, 9B), we performed Principal Component Analysis (PCA) using the variables fluorescence (area under the curve), length, area, roundness and perimeter of the Gastruloids within each experimental replicate (right panels of Figs. 7B, 8B, 9B). By comparing the degree of clustering between individual Gastruloids and their trajectories through the PCA plot, we were able to discern the subtle and major differences each experimental treatment had upon the patterning and morphology of the Gastruloids compared with the internal control (i.e. 48-72h Chi pulse). Each experimental replicate included this internal control for quality control and to provide a matched baseline for morphology 
bioRxiv preprint doi: https://doi.org/10.1101/051722; this version posted May 13, 2016. The copyright holder for this preprint (which was not certified by peer review) is the author/funder, who has granted bioRxiv a license to display the preprint in perpetuity. It is made available under aCC-BY 4.0 International license.

and Gastruloid progression.

In general, PCA of Gastruloids in control conditions revealed distinct clusters which corresponded to the time-point and the trajectories of these clusters through the plot were reproducible throughout replicate experiments (Figs. 7B, 8B, 9B, right panels). Gastruloids from early time-points were found to cluster tightly together (48h AA; red) with the final time-point (120h; white) being the most disperse; this may reflect heterogeneity between individual Gastruloids as they reach the end of their ability to be cultured for longer durations due to their increasing mass and propensity to adhere to the bottom of the wells at later times [15].

In all experiments (Figs. 2A-C, 7B, 8B, 9B) and in agreement with previous observations [13], at $48 \mathrm{~h}$ AA prior to the Chi pulse, a slight polarisation in the assumed-posterior region of the Gastruloids was observed which transiently increased in expression over time, peaking at $96 \mathrm{~h} \mathrm{AA} \mathrm{(Fig.} \mathrm{7B).} \mathrm{Importantly,} \mathrm{this}$ pattern of expression is highly reproducible and allows us to use their expression as a landmark for the effect of the treatment on the initial patterning event. Additionally, their reproducibility allows us to us to use the standard deviation within each experiment as a measure of the effect of the treatment. In the description of the data below, we refer to the treatment before the Chi pulse as pre-treatment.

\subsection{Nodal and FGF signalling are re- quired for the symmetry breaking event}

BMP signalling Pre-treatment with BMP4 did not alter the expression levels or localisation of T/Bra::GFP at 48h AA (Fig. 7B). However following the addition of Chi, BMP4 pre-treatment enhanced the expression of T/Bra::GFP throughout the whole Gastruloid compared to the control, although by $96 \mathrm{~h}$, this was rapidly down-regulated and the expression pattern was essentially identical to the control by $120 \mathrm{~h}$ AA (Fig. 7B, left panel). Progression of Gastruloid development following DMH1 pre-treatment was essentially identical to the control, although by $120 \mathrm{~h}$, DMH1 pre-treated Gastruloids were unable to maintain the expression of T/Bra::GFP (Fig. 7B, left panel). Whereas BMP4 pre-treatment showed a similar PCA distribution pattern to the controls over time with a slight increase in the spread in the second principal component at $48 \mathrm{~h}$ AA, DMH1 pre-treatment resulted in a less coherent grouping of individual Gastruloids as time progressed (Fig. 7C).

These data suggest that BMP signalling is not involved in the early patterning of the Gastruloids, although as shown by DMH1 pre-treatment, the presence of low levels at these early stages (Fig. 6) may impact the ability to sustain T/Bra::GFP expression at later time-points.
Nodal signalling Nodal pre-treatment (Fig. 7B) resulted in a similar fluorescence profile over time to that of the controls in terms of the fluorescence expression profile and the standard deviations between individual Gastruloids with no enhancement of the reporter at $48 \mathrm{~h}$, although, similar to BMP4 pretreatment, Nodal pre-treatment resulted in a global up-regulation of T/Bra::GFP at 72h (Fig. 7B, left panel). Inhibition of Nodal signalling with SB43 completely abolished the expression of T/Bra::GFP, where only a small proportion of Gastruloids showed any expression at 96-120h AA (Fig. 7B, left panel),, as well as a compromised pattern of growth, resulting in Gastruloids with a shorter final length. PCA revealed that although Nodal pre-treatment did not have a great effect on the overall trajectory of time-point clusters through the plot, there was a increase in the spread of the data points in both PC1 and PC2 at 48h AA (Fig. 7B, right panel). Inhibition of Nodal signalling with SB43 pre-treatment resulted in a complete disruption in the trajectories within the $\mathrm{PCA}$, reflecting the observations with the fluorescence analysis (Fig. 7B,C).

As the Gastruloids show such a distinct phenotype following Nodal inhibition prior to the Chi pulse, these data reveal the absolute requirement for Nodal signalling in the initial patterning of Gastruloids.

FGF/MAPK signalling To assess the role of FGF signalling, we pre-treated Gastruloids with either recombinant FGF2 or PD03 (MEK1/2 inhibitor) prior to the Chi pulse (Fig. 8A). Pre-treatment with FGF2 resulted in a $\mathrm{T} / \mathrm{Bra}: \mathrm{GFP}$ fluorescence profile with slightly increased heterogeneity between individual Gastruloids at $48 \mathrm{~h}$ AA compared with the vehicle control (Fig. 8B, left panel). By $72 \mathrm{~h}$ AA, the expression of T/Bra::GFP following FGF2 pre-treatment was essentially identical to the vehicle control, however, at 96h, FGF pre-treatment resulted in a higher maximum fluorescence than the control with the pole of expression less well defined (i.e. the expression was spread over a larger posterior region; Fig. 8B, left panel). This increased expression levels of the T/Bra::GFP reporter was maintained at $120 \mathrm{~h} \mathrm{AA}$ and although the region of expression had become more localised to the posterior region, the expression levels were augmented with respect to the controls (Fig. 8B, left panel). Interestingly, although Inhibition of FGF signalling by PD03 pre-treatment had little effect on the expression of T/Bra::GFP within the Gastruloids between 48 and $72 \mathrm{~h}$, Gastruloids at later time-points (96-120h AA) were severely retarded in their ability to up-regulate the reporter to the same extent as either the control or FGF2 pre-treatment condition (Fig. 8B, left panel). In both pre-treatment conditions, the gradual increase in the length of the T/Bra::GFP Gastruloids was not significantly altered over time when compared to the control(Fig. 8B, left panel).

In addition to the T/Bra::GFP cell line, we utilised an FGF reporter (Sprty4::H2B-Venus) mouse ESC line to assess FGF/MAPK signalling and assessed its expression following pre-treatment with either FGF2 or 
bioRxiv preprint doi: https://doi.org/10.1101/051722; this version posted May 13, 2016. The copyright holder for this preprint (which was

A

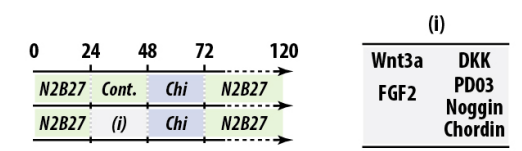

B

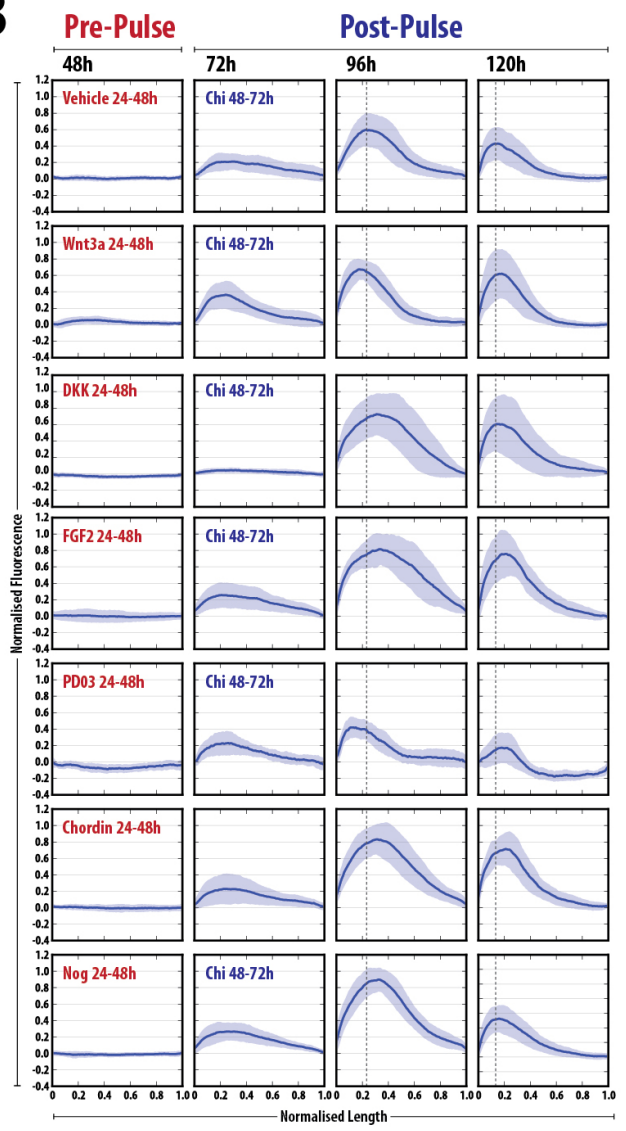

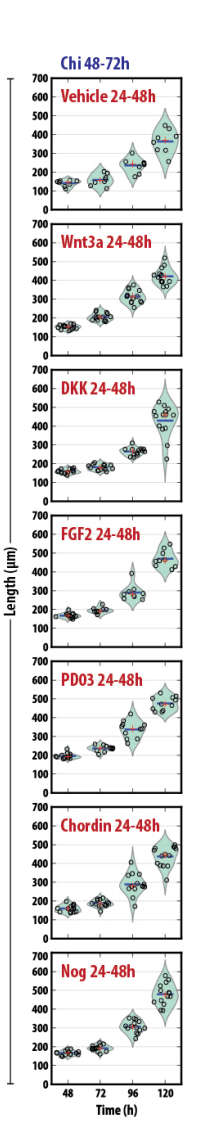

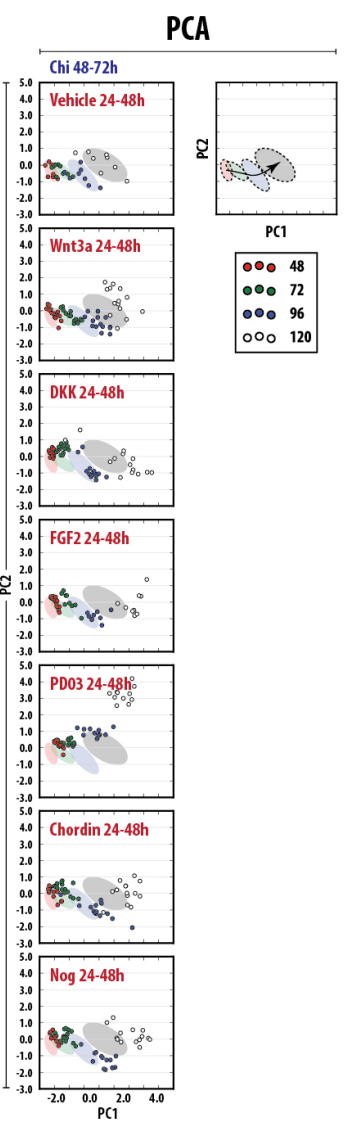

Figure 8: Effect of modulating Wnt, FGF and Nodal/BMP signalling prior to the Chi pulse. (A) Schematic representation of the experimental design (see Fig. 7 legend). (B,C) Individual Gastruloids treated as labelled were imaged by wide-field fluorescence microscopy and quantitatively analysed for the fluorescence (left panel), length (middle panel), and their Area, circularity, perimeter and roundness measured (not shown). Principal Component Analysis (right) was used to reduce the dimensions of the data, revealing distinct clusters which corresponded to the specific time-points. The fluorescence plots of the left of (B) and (C) were normalised to the maximum fluorescence of the control, and the maximum length of each Gastruloid was rescaled to 1 unit. Horizontal dashed lines at 72 and $96 \mathrm{~h} \mathrm{AA}$ in B indicate the maximum fluorescence of the Chi pulse condition.

PD03 (Fig. S2). Vehicle treatment resulted in a gradual increase in the FGF reporter over time with a slight bias towards the posterior region at 48 and $72 \mathrm{~h}$ AA (Fig. S2), with a burst of expression throughout the whole Gastruloids at $96 \mathrm{~h} \mathrm{AA}$. Interestingly, FGF2 pre-treatment did not have an effect on the average amplitude of the fluorescence between 48 and $72 \mathrm{~h}$ AA, although posterior pole of expression was more defined than the control, and treatment with PD03 resulted in a gradual decline of reporter expression over time (Fig. S2). By 96h Gastruloids pre-treated with FGF2 resulted in much higher levels at 96h than the control. Interestingly, the large increase and decrease in expression levels of the reporter following FGF2 or PD03 pre-treatment respectively occurred at the same time as the broadening and reduction (respectively) of the T/Bra::GFP. These data suggest that although
FGF signalling does not impact the initial patterning of the Gastruloids, it is involved in the maintenance of $\mathrm{T} / \mathrm{Bra}: \mathrm{GFP}$ expression at later stages.

Analysis of the Gastruloids using PCA revealed FGF2 pre-treatment to be broadly similar to the controls, with discrete clusters corresponding to the time interval following the same trajectory set out by the controls (Fig. 8B, right panel). However, FGF2 pretreatment resulted in a more rapid progression, where earlier time-points (e.g. 72h AA) occupied the regions reserved for the $96 \mathrm{~h}$ time-point (as defined by the controls; Fig. 8B, right panel). In addition, the heterogeneity between Gastruloids was greatly reduced and the clustering appeared tighter than the controls (Fig. 8B, right panel). Conversely, PD03 pretreatment greatly disrupted both the usual progression, indicating the requirement of FGF2 for correct passage 
bioRxiv preprint doi: https://doi.org/10.1101/051722; this version posted May 13, 2016. The copyright holder for this preprint (which was not certified by peer review) is the author/funder, who has granted bioRxiv a license to display the preprint in perpetuity. It is made available under aCC-BY 4.0 International license.

A

\begin{tabular}{|c|c|c|c|}
\hline N2B27 DMSO & Chi & $\mathrm{N} 2 \mathrm{~B} 2 \mathrm{~T}$ & IWP3 \\
\hline $\begin{array}{ll}N 2 B 27 & \text { (i) }\end{array}$ & Chi & N2B27 & XAV939 \\
\hline
\end{tabular}

B Pre-Pulse

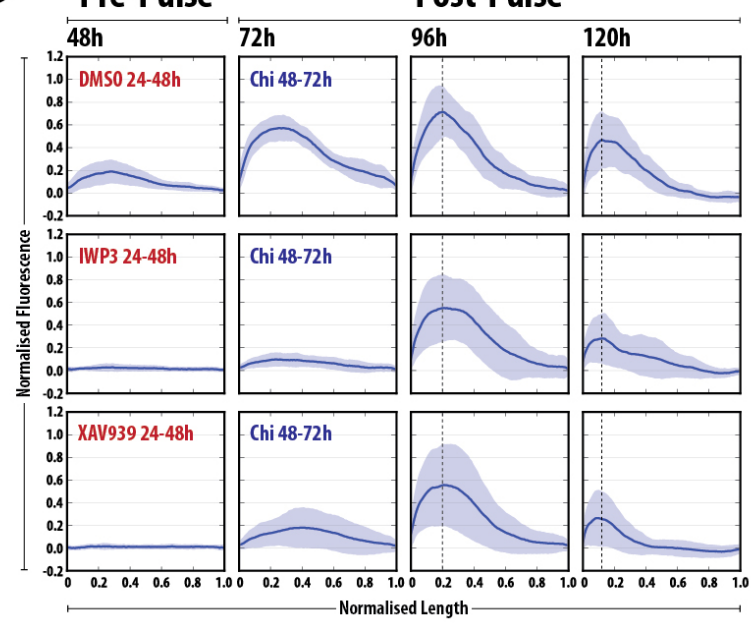

Post-Pulse

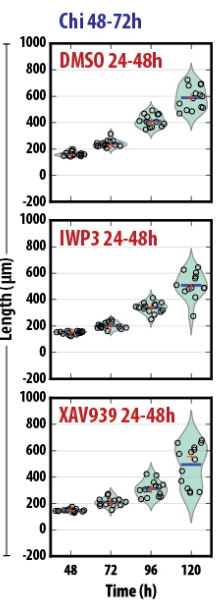

PCA

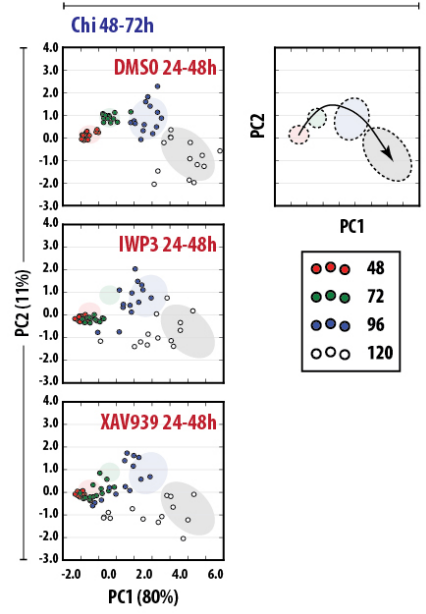

Figure 9: Quantitative analysis of T/Bra::GFP Gastruloids reveals defective pattern formation upon Wnt/ $\beta$ Catenin disruption between 24 and 48h. (A) A schematic of the experimental design for Gastruloid stimulation. Gastruloids, aggregated in N2B27 (green shading) were pulsed with either DMSO, the Porcupine inhibitor IWP3 or the Tankyrase inhibitor XAV939 between 24 and 48h AA. Following wash-off of these factors (see materials and methods), all Gastruloids were treated with a pulse of Chi (48-72h AA) followed by sustained culture in N2B27. Gastruloids were imaged by wide-field fluorescence microscopy and quantitatively analysed for the fluorescence (B, left panel), length (B, middle panel) and their Area, circularity, perimeter and roundness. Principal Component Analysis (B, right panel) revealed that the discrete clusters that corresponded to the time-point in DMSO conditions were heavily disrupted following IWP3 or XAV939 pre-treatment. The fluorescence in (B) was normalised to the maximum fluorescence of the DMSO control. In addition, the length of each Gastruloid was rescaled so the maximum length had a value of 1 unit. Horizontal dashed lines at 72 and $96 \mathrm{~h} \mathrm{AA}$ in B indicate the maximum fluorescence of the Chi pulse condition.

through the plot (Fig. 8B, right panel).

Taken together, these results indicate that FGF signalling has limited effect on the either initial patterning of the Gastruloid, the up-regulation of T/Bra::GFP fluorescence or progression of the Gastruloids, however the low, endogenous levels that are present at these early time-points are essential for constraining the domain of expression at the posterior region and the maintenance of increased expression at later timepoints. It may be the case that FGF signalling is acting to lower the threshold of the response.

Wnt/ $\beta$-Catenin signalling To assess the effect of Wnt/ $\beta$-Catenin signalling, we pre-treated Gastruloids with the recombinant proteins Wnt3a or DKK1, (Fig. 8) or small molecule inhibitors (IWP3: to inhibit the secretion of all Wnts [35]; XAV939; to increase $\beta$ Catenin degradation through tankyrase inhibition[36]; Fig. 9).

Treatment with Wnt3a resulted in a slightly enhanced posterior activation of $\mathrm{T} / \mathrm{Bra}: \mathrm{GFP}$ at $48 \mathrm{~h} \mathrm{AA}$ compared with the vehicle control (0.1\% BSA in PBS) which continued into the $72 \mathrm{~h}$ time-point (Fig. 8B). As time progressed, the Wnt3a pre-treated Gastruloids showed a narrower region of T/Bra::GFP expression, with the peak expression occurring more posteriorwise than the vehicle control, displaying less variation between individual Gastruloids (Fig. 8B). Although peak T/Bra::GFP expression occurred at $96 \mathrm{~h}$ AA following both vehicle and Wnt3a pre-treatment, Wnt3a tended to increase the fluorescence of the reporter to a greater extent than the control(Fig. 8B). Interestingly, whereas control Gastruloids generally reduced T/Bra::GFP expression by $120 \mathrm{~h}$, Wnt3a pre-treatment maintained the expression, and appeared to confine its expression to a narrower region within the posterior, albeit with a higher standard deviation(Fig. 8B).

Inhibition of LRP6-mediated Wnt signalling by pretreatment with recombinant Dkk1 resulted in an identical expression profile of T/Bra::GFP to vehicle control at 48h, however following Chi stimulation the fluorescence activation usually observed at $72 \mathrm{~h} \mathrm{AA}$ following vehicle or Wnt3 pre-treatment was abolished in the Dkk1-pre-treated Gastruloids (Fig. 8B). By 96h, the expression of the reporter had recovered, displaying maximum fluorescence at similar levels to the vehicle and Wnt3a conditions, however, the heterogeneity between individual Gastruloids was greatly increased 
bioRxiv preprint doi: https://doi.org/10.1101/051722; this version posted May 13, 2016. The copyright holder for this preprint (which was

and the polarisation was less defined (i.e. the fluorescence was less concentrated at the posterior region; Fig. 8B). After $120 \mathrm{~h}$ in culture, the fluorescence of Dkk1 pre-treated Gastruloids was broadly similar to the Wnt3a pre-treatment with a much higher degree of variation between individual Gastruloids than the vehicle control (Fig. 8B).

Pre-treatment with either IWP3 or XAV939 delayed the onset and magnitude of T/Bra::GFP expression in a similar manner to that of Dkk1 (Fig. 9B, left panel). In some replicate experiments in the XAV939-pre-treated Gastruloids, the fluorescence expression, although still generally located posteriorwise, displayed a greater degree of variation with respect to the DMSO control (data not shown). Similar to the Dkk1 pre-treated Gastruloids, by 96 and 120h AA, the pattern of T/Bra::GFP fluorescence was broadly similar to the controls although a greater deal of variation was observed between the Gastruloids pretreated with XAV939 and IWP3 and a slightly lower average expression level (Fig. 9B, left panel). Interestingly, Gastruloids pre-treated with IWP3 (which inhibits all Wnt secretion) was unable to maintain the expression of the reporter at $120 \mathrm{~h}$ compared with Dkk1 inhibition (inhibits at the level of LRP6), suggesting a requirement for non-canonical Wnts in the maintenance of T/Bra::GFP fluorescence.

Comparison of the lengths of the Gastruloids following either vehicle, Wnt3a or Dkk1 pre-treatment showed no differences in the progression of the average lengths, however Wnt3a pre-treatment reduced the heterogeneity between individual Gastruloids whereas Dkk1 pre-treatment resulted in a greater variation at $120 \mathrm{~h}$. However, there was a slight disruption in the maximum lengths obtained at $120 \mathrm{~h}$ AA following IWP3 or XAV939 pre-treatment, with a slightly lower average length and a larger spread of final lengths (Fig. 9B, right); this was more pronounced with XAV939 pre-treatment.

PCA analysis of Gastruloids pre-treated with Wnt3a and Dkk1 revealed similar trajectories to their control (Fig. 8B, 9B). Interestingly, Gastruloids pretreated with Wnt3a from the 72 and $96 \mathrm{~h}$ time-points were able to explore region usually reserved for the 96h and 120h time-points respectively (Fig. 8B, 9B), suggesting that Wnt pre-treatment may enhance the response to Chi. A number of subtle differences between the methods of Wnt inhibition by Dkk1, IWP3 or XAV939 pre-treatment was also revealed by the PCA analysis (Fig. 8B, 9B). Whereas pre-treatment with Dkk resulted in a similar trajectory to the control (although with a greater shift in the positive direction in PC1 at $72 \mathrm{~h} \mathrm{AA}$ ), the trajectories of IWP3 and XAV939 pre-treated Gastruloids was greatly disrupted compared to the controls and the data-points which correspond to specific time intervals were less coherently clustered (Fig. 9B). Prior to Chi stimulation, the clustering of the $48 \mathrm{~h}$ time-point of both IWP3 and XAV939 was dispersed along the $\mathrm{PC} 2$ to a greater extent than the control which was unable to be recovered after the Chi pulse (72h). By 96h, IPWP3 and
XAV939 pre-treated aggregates had largely recovered in terms of their positioning relative to the control, however XAV939 had not fully recovered and datapoints corresponding to individual Gastruloids were spread throughout PC1, occupying regions reserved for earlier time-points (Fig. 9B). After 120h in culture, neither IWP3 or XAV939 pre-treated Gastruloids were able to reach the same region of the PCA as the controls; this is reflected in the fluorescence traces which were unable to maintain T/Bra::GFP expression to the same extent as the control (Fig. 9B). These data suggest that the effects of IPW3 and XAV939 are greater than Dkk1-mediated Wnt inhibition, when many of the variables (length, fluorescence, roundness, area etc.) are taken into account. This further suggests a requirement of 'non-canonical' (i.e. $\beta$-Cateninand LRP6-independent) Wnt signalling at later stages, the secretion of which was inhibited by IWP3. Upon closer inspection of the expression of the T/Bra::GFP reporter within the Gastruloids, we found that whereas $\mathrm{T} /$ Bra::GFP expression is maintained at the elongating tip of the control Gastruloid (as previously described), treatment with IWP3 resulted the expression of the reporter along a mid-line region of a small subset of Gastruloids per experimental replicate (Fig. S3, indicated by white arrows); this phenotype was less pronounced in XAV939 pre-treatment conditions. Only one Gastruloid with Dkk pre-treatment showed this phenotype (data not shown).

Section summary Although there was slight variation between replicates (in terms of expression levels and the internal standard deviations), these observations indicate that whereas BMP and FGF signalling has minimal effect at the onset of the initial patterning event, Nodal signalling is absolutely essential to allow the not only the expression of T/Bra::GFP but possibly its correct placement within the aggregate and $\mathrm{Wnt} / \beta$ Catenin signalling is required in this early period (24$48 \mathrm{~h} \mathrm{AA}$ ) for the correct timing of expression. The requirement of BMP signalling later in the Gastruloid development suggests two fundamental stages of Gastruloid development: one base on establishing the pattern which is Nodal and Wnt dependent and the second on its growth, and elongation involving a late requirement of both FGF2 and BMP signalling, and 'noncanonical' (e.g. LRP6-independent) Wnt signalling. In addition, these results clearly demonstrate the reproducibility of the Gastruloid phenotype not only within experiments but throughout replicate experiments; this measure of bother intra- and inter-experimental reproducibility has not to our knowledge been demonstrated other organoid culture techniques [37].

\section{Discussion}

In the mouse, the anteroposterior axis is established early in the cup-shaped zygote through the localisation of Nodal, BMP and Wnt signalling to a proximoposterior region of the embryo that becomes the 
bioRxiv preprint doi: https://doi.org/10.1101/051722; this version posted May 13, 2016. The copyright holder for this preprint (which was

posterior pole, where gastrulation is initiated as reflected in the expression of T/Bra (Fig. 1). Here we have shown that Gastruloids, embryonic organoids derived from small aggregates of mouse ESCs, undergo symmetry-breaking and gene expression polarisation in a manner that mirrors events in embryos [13-15, 37]. However, in contrast with embryos that use a sequence of interactions between extraembryonic and embryonic tissues to pattern themselves, Gastruloids are composed only of embryonic tissue and reveal an intrinsic capacity for axial organisation that is driven by interactions between Nodal and Wnt signalling. Analysis of signalling reporters in the Gastruloids reveals clear Wnt/ $\beta$-Catenin and Nodal/Smad2/3 activity from 24 $48 \mathrm{~h}$ AA which often becomes localised to one pole of the aggregate and correlates with the onset and localisation of $\mathrm{T} / \mathrm{Bra}$ expression. Furthermore, as in the embryo, this pattern of $T / B r a$ expression is dependent on Nodal and Wnt signalling: exposure of Gastruloids to inhibitors of these pathways between 24 and $48 \mathrm{~h} \mathrm{AA}$ abolishes or reduces these patterns.

The absence of extraembryonic tissues is reflected in the absence of expression of Trophoectoderm and Visceral Endoderm markers $(B M P 4$, Cerberus or Dkk1) and the absence of BMP reporter activity (Figs. 3, 6). Furthermore, addition of BMP to the culture, has little effect on the patterning process other than a transient increase in the levels of $\mathrm{T} / \mathrm{Bra}$ through alterations of Nodal and Wnt signalling (data not shown). In the embryo, BMP is deployed in the Extraembryonic Ectoderm and regulates the expression of Nodal and Wnt3 in the proximal part of the embryo [38, 39]; a similar effect has been reported in differentiating ESCs [40, 41] and can account for the observed effect BMP on the Gastruloids.

The strict dependence of Nodal and Wnt expression on BMP signalling in the embryo and the requirement for their antagonists in the symmetry-breaking event, raise questions about the molecular origin of polarity in our experiments. In the embryo Nodal plays a key role in the establishment of AP polarity $[42,43]$ and this also appears to be the case in the Gastruloids where in the absence of Nodal signalling there is neither localisation of T/Bra::GFP expression nor clear polarisation of the Gastruloid. Furthermore, in the embryo successive patterns of Nodal expression outline the emergence of the AP axis: it is first expressed throughout the E4.5 blastocyst and the E5.5 epiblast, where it then becomes restricted, first to its proximo-posterior region and shortly afterwards, in a Wnt/ $\beta$-Catenin signalling dependent manner, to the Primitive Streak [44]. The patterns of Nodal expression in the Gastruloids are reminiscent of these: an onset of low ubiquitous expression coincident with the extinction of Nanog expression, a Wnt/ $\beta$-Catenin dependent rise between 48 and $72 \mathrm{~h} \mathrm{AA}$ and then a restriction to the elongating region (Fig. 4). These patterns of Nodal expression are associated with Smad2/3 activity (Fig. 5). Interestingly, around $96 \mathrm{~h} \mathrm{AA}$ we observe the emergence of a spot of Nodal expression at the anterior border of the elongating region which could correspond to a struc- ture similar to the node in the embryo $[45,46]$. This possibility is supported by the observation of Smad2/3 reporter activity between 96 and $120 \mathrm{~h} \mathrm{AA}$ on one side of the Gastruloid (see Fig. 4, 5); in some instances it is possible to see a similar but faint pattern of Nodal expression (data not shown). This pattern of activity is reminiscent of the Left-Right asymmetry expression of Lefty and Nodal in the embryo [47] and suggests that the Gastruloids not only develop an AP axis but also exhibit bilateral asymmetry.

The self organised patterning of the Gastruloids is, at first sight, surprising but might reflect a situation, latent in the embryo, which generates asymmetries in gene expression within the E5.0 Epiblast, before the restriction of primitive streak initiation to the proximal posterior part of the embryo $[48,49]$. This possibility is supported by some observations. For example, the epiblast of embryos double mutant for Cerl and Lefty1 is not unpatterned and exhibits a residual asymmetry in Nodal expression and, sometimes, double axes [49, 50]; this suggests that the epiblast can pattern itself in the absence of the extraembryonic repressors. Similarly, loss of $D k k 1$ and gain of function $\beta$-Catenin do not alter the axial organisation of the epiblast, though they eliminate the head [51]. It could be argued that there are functional redundancies in the antagonists of Wnt and Nodal signalling and that this explains the partial polarity in Dkk and Cerb/lefty double mutants. However, this does not account for the observed phenotypes and suggests that these antagonisms are not drivers of AP patterning.

Our results argue for an independent and intrinsic self-patterning ability in the epiblast, and we would like to suggest that the role of the visceral endoderm and the extraembryonic ectoderm (trophoectoderm) is not to break the initial symmetry but to bias and stabilise an intrinsic symmetry-breaking event in the epiblast. Crucially, to position the initiation of gastrulation precisely and reproducibly to one end of the proximal part of the conceptus: near the extraembryonic Ectoderm. The notion that within organisms, intrinsic patterning activities of individual structures are biased and contribute in these complex connected structures has been discussed in other related contexts [37]. The reason for this is likely to lie in the fact that the first cells to leave the Primitive Streak will allocate themselves within the Extraembryonic Ectodermal territory to give rise to the allantois and the chorion [52,53], and that positioning the initiation of gastrulation to the proximal domain of the embryo facilitates these cells to find their target. A process solely determined by a spontaneous symmetry-breaking event would position this point indiscriminately and therefore not contribute efficiently to the general organisation and structure of the conceptus. Thus, it is possible that the interactions between embryonic and extraembryonic tissues during axial specification are related to the interactions between the embryo and the mother more than between the organism and the embryo. 
bioRxiv preprint doi: https://doi.org/10.1101/051722; this version posted May 13, 2016. The copyright holder for this preprint (which was

Nodal signalling and AP polarity Our results suggest that symmetry-breaking in Gastruloids is reliant on Nodal that is expressed from the moment of aggregation. Nodal is known to play a critical role in the establishment of the AP axis in all vertebrates $[54,55]$ and, together with its transcriptional target and feedback inhibitor Lefty1, has been shown to have an intrinsic symmetry-breaking ability [56-58]. Our results suggest that it is this characteristic that probably mediates the symmetry-breaking in the Gastruloids. We observe low levels of Nodal signalling as soon as the aggregate forms and the expression of Nanog, a pluripotency marker, is extinguished. This has been observed in ESCs [59] and might reflect the switch between pluripotency and differentiation. Later, between 24 and $48 \mathrm{~h} \mathrm{AA}$ we detect a range of patterns of the Smad2/3 reporter, with frequent small patches of high levels of activity polarised to one side. We also detect low levels of Lefty1 expression, which is a target of Nodal and its main partner of its self patterning capacity. Furthermore, Nodal is a target of Nodal signalling [57] and also has an input from Wnt signalling [60]; this is also recapitulated here where Wnt/ $\beta$-Catenin signalling does lead to an increase in Nodal expression (Fig. 4). At $48 \mathrm{~h} \mathrm{AA}$, we observe patches of low level T/Bra::GFP expression and Wnt/ $\beta$-Catenin signalling. In the absence of any extrinsic signals, this pattern evolves and by $72 \mathrm{~h} \mathrm{AA}$ it is possible to observe T/Bra::GFP and Wnt/ $\beta$-Catenin signalling localised to one pole and Nodal expression to the elongating region in a variable domain of the initial Gastruloids. In the embryo this pattern of expression marks the start of gastrulation at E6.2 and therefore, is consistent with our estimate that $48 \mathrm{~h} \mathrm{AA}$ is about E5.5 as this maps the interval between 48 and $72 \mathrm{~h} \mathrm{AA}$ to approximately between E5.5 and E6.5 (Fig. 10).

In the embryo, expression of Nodal at E5.5 appears heterogeneous (see Fig. 1A in [44]) as it is at $48 \mathrm{~h} \mathrm{AA}$ in our experiments. This pattern and its evolution might represent the development of a Reaction-Diffusion ( $\mathrm{R}$ D) system that drives the symmetry-breaking event both, in the embryo and the Gastruloid [56, 57, 61]. The dependence of an intrinsic symmetry-breaking event on Nodal signalling in the embryo is supported by the observation that the patterning defects observed in Cerberus, Lefty double mutants are corrected by reducing the dosage of Nodal in the mutant embryos [49]. It may be that the interactions between the extraembryonic and embryonic tissues raise the threshold for Nodal activity in the embryo and that this is part of the proximodistal biasing mechanism that we have discussed. The notion that interactions between tissues change the thresholds of signalling events involved in pattern formation in order to positions structures relative to each other might be a principle that emerges from these and related studies [37].

In the embryo, Nodal signalling requires Furin convertase activity which, during E5.0 and E6.0 is provided by the extraembryonic ectoderm [60]. In principle this activity should be missing in the Gastruloids but, as they pattern themselves in a Nodal dependent manner and we observe Nodal dependent activity of a Smad2/3 reporter, there must be some residual convertase activity or, as has been suggested, the Nodal precursor is able to signal through Activin receptors [38]. Further work should clarify this.

The role of Wnt signalling The establishment of the AP axis in vertebrate embryos is marked by the onset of gastrulation (represented by the primitive streak in amniotes) at the posterior midline . Experiments in Xenopus revealed that, in addition to Nodal, Wnt/ $\beta$-Catenin signalling plays a key role in this process [63-65], and similar requirement has been revealed in chicken embryos, where the initiation of gastrulation requires a synergy between Nodal and Wnt/ $\beta$-Catenin signalling [66, 67]. Analysis of mouse mutants for elements of Wnt signalling supports this interaction $[7,8]$ : while gain of function of Wnt signalling can generate an ectopic axis $[68,69]$, loss of function leads to defects in polarity and axial extension [70]. Nodal and Wnt signalling synergise in the induction of $T / B r a$ expression and the initiation of the primitive streak $[66,67]$ but, how interactions between these signalling pathways lead to a robust AP axis remains open to discussion. Our experiments provide some insights into the nature of these interactions.

At $48 \mathrm{~h} \mathrm{AA}$, the patterning of Gastruloids appears to be a stochastic event. Whether a Gastruloid will elongate or not in the next 48 hours appears to correlate with the localisation of $\beta$-Catenin/TCF, Smad2 $/ 3$ signalling and $\mathrm{T} / \mathrm{Bra}$ expression to one pole of the Gastruloid, and varies from experiment to experiment. Surprisingly, exposure to Chi or Wnt3a for $24 \mathrm{~h}$ between 48 and $72 \mathrm{~h} \mathrm{AA}$ leads to an almost uniform polarisation of both T/Bra::GFP and Wnt signalling as well as elongation with $\mathrm{T} / \mathrm{Bra}: \mathrm{GFP}$ at the tip of the Gastruloid across a population (Fig. 2, 7). This observation is surprising as there is no polarised exposure to Chi or Wnt and yet, the response is localised and triggers a precisely spatially orientated event. Furthermore, time-lapse imaging of the behaviour of Gastruloids during and after exposure to Chi reveals a transient global response in the form of ubiquitous expression of T/Bra::GFP, which then relaxes to the posterior region of the Gastruloids, to where is was before the signalling pulse (Fig. 2D). It seems as if the only region able to respond would be the one that had been already chosen.

There are several examples in which ubiquitous tonic activation of Wnt signalling results in a localised response or, in many instances, is capable of rescuing a Wnt signalling mutants [71-76]. Furthermore, loss of Wnt signalling reduces but does not abolish the expression of genes under its control $[70,77]$ and in some instances, has no effect on the pattern [78, 79]. One explanation for these observations is that rather than working as a morphogen or directing the output of a gene regulatory network (GRN), Wnt signalling controls the signal to noise ratio of the processes it is associated with $[75,76]$. In the case of the Gastruloids, this would be the Nodal-driven symmetry-breaking process 


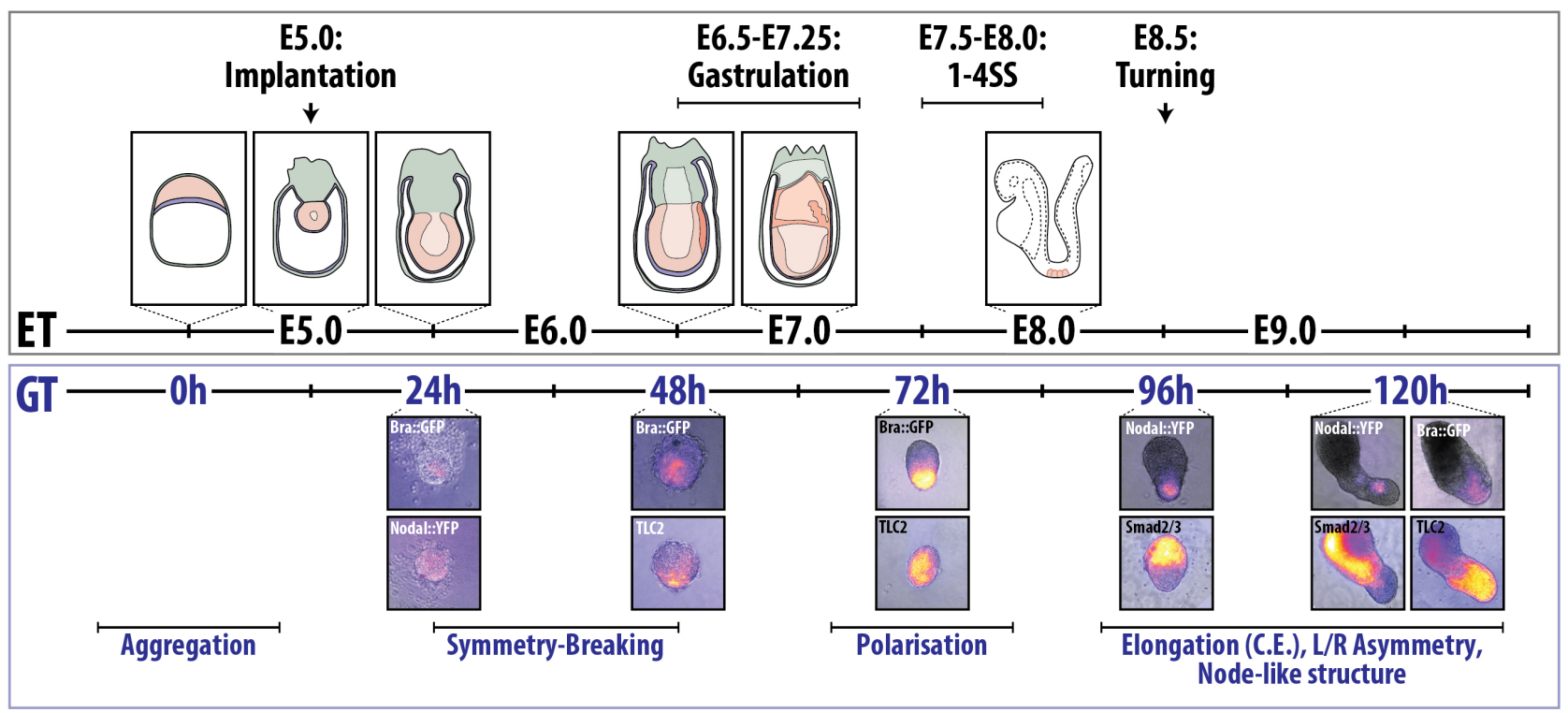

Figure 10: Alignment of the developmental stages of the mouse Embryo and Gastruloids. Top panel indicates Embryo Time (ET), displaying schematic representations of the mouse embryo with key developmental landmarks. Bottom panel indicates Gastruloid Time (GT) with images of Gastruloids at indicated time-points expressing the reporter constructs described in this study, aligned to the approximate developmental stage of the embryo ( \pm 0.5 days). Figure part adapted from [8] and the e-Mouse Atlas Project's Theiler Stages website resource [62].

and its effect on $\mathrm{T} /$ Bra which is a target of $\mathrm{Wnt} / \beta$ Catenin signalling. In support of this, exposure to the Nodal/ALK4/7 signalling inhibitor SB43 during 24-48h AA abolishes the response to Chi and the polarisation of the aggregate. Furthermore, increases in Wnt/ $\beta$-Catenin, but not Nodal, signalling will rescue losses of Wnt signalling during the same period. This suggests that Nodal is the main driver of the symmetrybreaking event during 24-48h AA and that Wnt/ $\beta$ Catenin signalling modulates its effects.

Our results support the hypothesis that a key role of Wnt signalling in developmental events is to act as a filter in cell fate decisions that regulates the ratio of signal to noise of specific processes $[75,76,80]$ and suggest that in the Gastruloids, the effect of Wnt/ $\beta$ Catenin signalling is to stabilise the molecular and cellular events that have taken place during 24-48h AA, principally the activity of Nodal/Smad2/3 activity. Interestingly, application of Chi after disrupting Wnt signalling during this period results in a process with much more variability than the wild type situation (Fig. 9). One interpretation of this observation is that, in the absence of Wnt/ $\beta$-Catenin signalling, the output of Nodal/Smad2/3 activity is noisy and this is what is amplified by Chi between 48 and $72 \mathrm{~h}$ AA i.e. Wnt/ $\beta$-Catenin signalling acts as an amplifier/filter of previous events rather than the driver of the symmetrybreaking, which is likely to be Nodal. This situation is supported by the phenotype of Wnt3 mutant embryos which exhibit initial, weak but localised expression of $\mathrm{T} /$ Bra that then progresses (or not) in a stochastic manner [81, 82].
Temporal correspondence between events in Gastruloids and embryos The sequence of transcriptional and signalling events that we have described allows us to align the patterning of the Gastruloids with that of embryos and refine a previous estimate for the relative timing of events [13]. We surmise that the period between 48 and $72 \mathrm{~h} \mathrm{AA}$ corresponds to E5.5E6.5 in embryos and serves to anchor our time-line (Fig. 10). This anchorage is corroborated by the patterns of expression of Nodal, FGF4, 5, Wnt3 and Wnt3a that mirror the events in the embryo during this time period [83, 84] (Fig. 3). Furthermore, we observe re-expression of Nanog in the elongate at $72 \mathrm{~h}$ AA (Fig. 4E), something that has also been described in the embryo in the nascent mesoderm at the start of Gastrulation [85]. It is in this period that AP polarity is firmly established, $\mathrm{T} / \mathrm{Bra}$ expression becomes restricted to the proximal posterior region and gastrulation begins [82] and we see analogous events in the Gastruloids (see also van den Brink et al. [13]). The emergence of a node like structure at around $96 \mathrm{~h}$ makes this time-point equivalent to E7.5 and the emergence of bilaterally asymmetric signalling associated with this structure shortly afterwards buttresses this comparison. Altogether these observations suggest a surprisingly close timing of events and of regulatory interactions between the Gastruloids and embryos that is summarised in Fig. 10.

Symmetry breaking Gastruloids and in mouse embryos Our results show that between 24 and 48h AA, Gastruloids undergo a Nodal dependent symmetry-breaking event that, in collaboration with Wnt signalling, leads to the robust polarised expression 
bioRxiv preprint doi: https://doi.org/10.1101/051722; this version posted May 13, 2016. The copyright holder for this preprint (which was

of T/Bra::GFP to one pole which, by comparison with the embryo, we define as the posterior. We surmise that a similar symmetry-breaking event also occurs in the embryo around E5.5 but exploration of this possibility is constrained by the difficulties of working with embryos at this early stage. However, our system allows detailed analysis of the relationship between the different signalling and transcription factor networks which help enlighten these early stages. The suggested correspondence of events suggests that the period between 24 and $48 \mathrm{~h} \mathrm{AA}$ corresponds to between E4.5 and E5.5 and that this period is important for the establishment of the AP axis. While there is evidence for early engagement of Nodal in this process [42-44, 86], our results suggest an involvement of Wnt signalling in AP axis specification which has not been reported before. For example, inhibition of WNT signalling inhibition of Wnt signalling, e.g. with Porcupine mutants, shows no effect in the patterning of the embryo until the onset of gastrulation [87, 88]. This could be interpreted to suggest that the events that we observe in our system are not informative of the events in vivo. However, elements of Wnt signalling are expressed in the embryo from the blastocyst stage [89] and gain and loss of $\beta$-Catenin function, as well as of non canonical Wnt signalling, have effects earlier than those of Porcupine mutants [8]. Therefore, there might be a Wnt independent $\beta$-Catenin signalling event early in development that affects AP axis formation. We suggest that this is the case and that it is these activities that are revealed in our experiments. Our experimental system provides an opportunity to study this further.

Our results also highlight an important role for FGF signalling in the symmetry-breaking events. Inhibition of MEK signalling compromises the patterning of the Gastruloids in a manner similar to, but not as extreme as, that resulting from inhibition of Nodal signalling: loss of polarity, elongation and T/Bra::GFP expression. As in the case of Nodal signalling, this defect is not rescuable by Chi. On the other hand, exposure to FGF during 24-48h AA increases the domain of T/Bra::GFP expression and interferes, albeit temporarily, with elongation. In the embryo, it is often suggested that FGF signalling is required for the transition from the blastocyst to the epiblast [90-93] but analysis of $F G F 4,5,8$, and FGF receptor mutants has not revealed any role in axis establishment though there are clear defects associated with mesoderm establishment and axial elongation [94-96]. Furthermore, exposure of early embryos to FGF leads to an expansion of the T/Bra::GFP expression domain [97] as we observe here (Fig. 8) and is consistent with a role in the onset of gastrulation.

As in the case of Wnt signalling, our results suggest a role of FGF signalling in the symmetry-breaking events which might also be operative but difficult to study in the embryo. Our study also shows that FGF/ERK signalling is closely associated with Wnt/ $\beta$ Catenin signalling in the induction of $\mathrm{T} / \mathrm{Bra}$ expression and therefore in the establishment of the Primitive Streak, and that this might be through modulation of the signalling event or through the target [98]. A related function of FGF in mesoderm induction has been described in other vertebrates $[99,100]$ but this is the first clear evidence that this might also be the case in mammals where it is often more closely associated with the transition from pluripotency to differentiation.

Anteroposterior patterning in Gastruloids and embryos A significant feature of the Wnt/ $\beta$-Catenin driven patterning of the Gastruloids is the absence of proneural gene expression in the anterior domain [13]. When maintained in N2B27 many Gastruloids will develop Sox1-expressing cells characteristic of neural tissue $[13,14]$ and this default anterior neural fate is the basis for protocols to generate forebrain and eye cup structures [101-104]. However, after a pulse of Wnt/ $\beta$ Catenin signalling, Gastruloids are able to generate spinal cord-like tissue because they are able to create Neuromesodermal progenitors, the pool that gives rise to this posterior structure and the paraxial mesoderm [14]. This independence of anterior and posterior development reflects the situation in the embryo where both regions are specified through separate mechanisms and, probably, at different times $[105,106]$. This can be observed in, for example, loss of Dkk1 activity or in mutations resulting in a gain of $\beta$-Catenin $[51,107,108]$ that produce embryos lacking anterior structures, reminiscent of Gastruloids after exposure to high levels of Wnt/ $\beta$-Catenin signalling. It is noteworthy that gain of function constitutive mutations in the Wnt signalling receptor LRP6 or in $\beta$-Catenin, although extend the domain of Wnt signalling anteriorly, never cover the whole territory and leave a domain at the anterior most region of the embryo unresponsive [51]. This situation is also very similar to the one Gastruloids experience when they are exposed to high levels of Wnt signalling in which there is a transient ubiquitous response but in the anteriormost region the response is not stabilised.

Altogether, these observations suggest that when the AP axis is established the primitive streak specifies mesodermal and endodermal derivatives but that the anterior region of the embryo, that will give rise to the brain, is protected from this fate and delayed in its differentiation. The early suppression of BMP, Nodal and Wnt signalling does not lead to neural fate but rather to a pre-neural state, perhaps simply an epiblast state, protected from becoming primitive streak $[109,110]$. This region becomes neural upon the release of antagonists of BMP and Nodal signalling from the prechordal plate and anterior definitive endoderm (ADE) at the end of gastrulation (reviewed in [111]). In a mirror image event, loss of BMP or Nodal signalling leads to embryos with, mostly, anterior neural structures $[86,112]$. Failure to generate prechordal plate or the ADE leads to anterior truncations which can be compared to what we observe in our experiments $[113,114]$.

There is a clear and early subdivision of the aggregate into two domains that will respond differently to signals: Wnt/ $\beta$-Catenin signalling during 48 and $72 \mathrm{~h}$ 
AA stabilises the expression of $\mathrm{T} / \mathrm{Bra}$ in the posterior region but it induces Nodal expression throughout the aggregate and, furthermore, there is a transient ubiquitous expression of $\mathrm{T} / \mathrm{Bra}$ as well as of the $\mathrm{Wnt} / \beta$ Catenin reporter expression that then relaxes to the pre-pulse situation. As transiently there are high levels of Nodal and Wnt/ $\beta$-Catenin signalling in the anterior domain and there is no expression of $\mathrm{T} / \mathrm{Bra}$, this suggests that the symmetry-breaking event not only leads to a posterior like domain that will develop as in the embryo, but also that it generates an anterior domain that is refractory to this signalling event and upon which Wnt acts. The expression of high levels of the Smad2/3 reporter in this region confirm the increase in Nodal as well as highlight how this domain is refractory to $\mathrm{T} / \mathrm{Bra}$ expression. On the other hand, this domain of Nodal signalling, provide an explanation for why this domain is also refractory to the neural fate as Nodal/Smad2/3 signalling will protect this domain from neural development. This suggests that Gastruloids have an anterior domain that is refractory to posterior development. It may be in this primed state and will be timely localised provision of BMP and Nodal inhibitors that releases this potential.

Conclusions Our results suggest that the symmetry-breaking event in the mouse embryo results from the self-organising activity of Nodal signalling around E5.0 which is amplified and stabilised at the proximal-posterior region of the embryo by interactions with agonists and antagonists of BMP, Wnt and Nodal itself, between E5.5 and E6.0. These interactions are patterned and lead to localisation of the initiation of gastrulation to a strategic point that allows the first cells that leave the Primitive Streak to invade the Extraembryonic Ectoderm. In the embryo it is likely that the threshold for this is raised by the extraembryonic tissue to ensure the role and outcome of the bias. Probably because of their characteristics, Gastruloids reveal and allow the amplification of this event. We have suggested a similar explanation of a related situation in the patterning of the neural tube in ESC-derived cysts [37]; perhaps there is a principle behind this which should be borne in mind in the development of tissues and organs in culture which may follow different paths than in embryos where their development will be constrained by the need to place them relative to other tissues and organs.

Our results show that the symmetry-breaking events in Gastruloids, an embryonic organoid system, recapitulate the main events in the embryo. Furthermore, their development closely mirrors that of embryos and allows us to position landmarks for an experimental time-line. Because of the ease of their manipulation, Gastruloids represent a good experimental system to explore the mechanisms underlying early developmental events. Here we have used them to reveal novel requirements and interactions for Wnt, Nodal and FGF signalling for symmetry-breaking that are extrapolatable to embryos.

There are other systems in which ESCs can be spatially patterned $[115,116]$ and each of them can make their own contribution to our understanding of the connection between cell fate assignments and the three dimensional organisation of tissues and organs. The observations that we report here on Gastruloids, particularly their ability to be cultured long term and the observation that in addition to an AP axis they can develop an LR asymmetry and generate a node-like structure, suggest that they might be useful beyond the early stages of development.

Acknowledgements We thank G. Keller for the T/Bra::GFP line, A-K Hadjantonakis for the TLC2 reporter and members of the AMA lab for useful discussions and criticisms. This work is funded by a European Research Council (ERC) Advanced Investigator Award to AMA (DAT, PCH) with the contribution of a Project Grant from the Wellcome Trust to AMA, an Engineering and Physical Sciences Research Council (EPSRC) Studentship to PB-J. 
bioRxiv preprint doi: https://doi.org/10.1101/051722; this version posted May 13,2016. The copyright holder for this preprint (which was not certified by peer review) is the author/funder, who has granted bioRxiv a license to display the preprint in perpetuity. It is made available under aCC-BY 4.0 International license.

\section{References}

[1] S Roth and J A Lynch. Symmetry Breaking During Drosophila Oogenesis. Cold Spring Harbor Perspectives in Biology, 1(2):a001891-a001891, August 2009. doi: 10.1101/cshperspect.a001891. URL http://cshperspectives.cshlp.org/lookup/doi/10.1101/cshperspect.a001891.

[2] V Riechmann and A Ephrussi. Axis formation during Drosophila oogenesis. Current Opinion in Genetics 83 Development, 2001. URL http://www.sciencedirect.com/science/article/pii/ S0959437X00002070.

[3] Katsuyoshi Takaoka and Hiroshi Hamada. Cell fate decisions and axis determination in the early mouse embryo. Development, 139(1):3-14, January 2012. doi: 10.1242/dev.060095. URL http://eutils.ncbi. nlm.nih.gov/entrez/eutils/elink. fcgi?dbfrom=pubmed\&id=22147950\&retmode=ref\&cmd=prlinks.

[4] Federica Bertocchini and Claudio D Stern. The hypoblast of the chick embryo positions the primitive streak by antagonizing nodal signaling. Developmental Cell, 3(5):735-744, November 2002. URL http://eutils.ncbi.nlm.nih.gov/entrez/eutils/elink.fcgi?dbfrom=pubmed\&id=12431379\& retmode=ref\&cmd=prlinks.

[5] J Rossant and P P L Tam. Blastocyst lineage formation, early embryonic asymmetries and axis patterning in the mouse. Development, 136(5):701-713, February 2009. doi: 10.1242/dev.017178. URL http: //dev.biologists.org/cgi/doi/10.1242/dev.017178.

[6] Claudio D Stern. Evolution of the mechanisms that establish the embryonic axes. Current Opinion in Genetics 83 Development, 16(4):413-418, August 2006. doi: 10.1016/j.gde.2006.06.005. URL http: //linkinghub.elsevier.com/retrieve/pii/S0959437X06001146.

[7] Jaime A Rivera-Pérez and Anna-Katerina Hadjantonakis. The Dynamics of Morphogenesis in the Early Mouse Embryo. Cold Spring Harbor Perspectives in Biology, 7(11), November 2015. doi: 10.1101/cshperspect.a015867. URL http://eutils.ncbi.nlm.nih.gov/entrez/eutils/elink.fcgi? dbfrom=pubmed\&id=24968703\&retmode=ref\&cmd=prlinks.

[8] Silvia Muñoz-Descalzo, Anna-Katerina Hadjantonakis, and Alfonso Martinez Arias. Wnt/B-catenin signalling and the dynamics of fate decisions in early mouse embryos and embryonic stem (ES) cells. Semin Cell Dev Biol, August 2015. doi: 10.1016/j.semcdb.2015.08.011. URL http://eutils.ncbi.nlm.nih. gov/entrez/eutils/elink.fcgi?dbfrom=pubmed\&id=26321498\&retmode=ref\&cmd=prlinks.

[9] Daniel St Johnston. THE ART AND DESIGN OF GENETIC SCREENS: DROSOPHILA MELANOGASTER. Nat Rev Genet, 3(3):176-188, March 2002. doi: 10.1038/nrg751. URL http: //www. nature.com/doifinder/10.1038/nrg751.

[10] Kathryn V Anderson. Finding the genes that direct mammalian development. Trends in Genetics, 16(3): 99-102, March 2000. doi: 10.1016/S0168-9525(99)01921-6. URL http://linkinghub.elsevier.com/ retrieve/pii/S0168952599019216.

[11] Hiroshi Hamada. Role of physical forces in embryonic development. Semin Cell Dev Biol, 47-48:88-91, December 2015. doi: 10.1016/j.semcdb.2015.10.011. URL http://linkinghub.elsevier.com/retrieve/ $\mathrm{pii} / \mathrm{S} 1084952115002086$.

[12] R Hiramatsu, T Matsuoka, C Kimura-Yoshida, and S W Han. External Mechanical Cues Trigger the Establishment of the Anterior-Posterior Axis in Early Mouse Embryos. Developmental Cell, 2013. URL http://www.sciencedirect.com/science/article/pii/S1534580713005741.

[13] Susanne C van den Brink, Peter Baillie-Johnson, Tina Balayo, Anna-Katerina Hadjantonakis, Sonja Nowotschin, David A Turner, and Alfonso Martinez Arias. Symmetry breaking, germ layer specification and axial organisation in aggregates of mouse embryonic stem cells. Development, 141(22):4231-4242, November 2014. doi: 10.1242/dev.113001. URL http://eutils.ncbi.nlm.nih.gov/entrez/eutils/ elink. fcgi ?dbf rom=pubmed\&id=25371360\&retmode=ref\&cmd=prlinks.

[14] David A Turner, Penelope C Hayward, Peter Baillie-Johnson, Pau Rué, Rebecca Broome, Fernando Faunes, and Alfonso Martinez Arias. Wnt/ $\beta$-catenin and FGF signalling direct the specification and maintenance of a neuromesodermal axial progenitor in ensembles of mouse embryonic stem cells. Development, 141(22):4243-4253, November 2014. doi: 10.1242/dev.112979. URL http://eutils.ncbi.nlm. nih.gov/entrez/eutils/elink.fcgi?dbfrom=pubmed\&id=25371361\&retmode=ref\&cmd=prlinks. 
bioRxiv preprint doi: https://doi.org/10.1101/051722; this version posted May 13, 2016. The copyright holder for this preprint (which was not certified by peer review) is the author/funder, who has granted bioRxiv a license to display the preprint in perpetuity. It is made available under aCC-BY 4.0 International license.

[15] Peter Baillie-Johnson, Susanne Carina van den Brink, Tina Balayo, David Andrew Turner, and Alfonso Martinez Arias. Generation of Aggregates of Mouse Embryonic Stem Cells that Show Symmetry Breaking, Polarization and Emergent Collective Behaviour In Vitro. J Vis Exp, (105), 2015. doi: 10.3791/53252. URL http://eutils.ncbi.nlm.nih.gov/entrez/eutils/elink.fcgi?dbfrom= pubmed\&id=26650833\&retmode=ref\&cmd=prlinks.

[16] Hans Jörg Fehling, Georges Lacaud, Atsushi Kubo, Marion Kennedy, Scott Robertson, Gordon Keller, and Valerie Kouskoff. Tracking mesoderm induction and its specification to the hemangioblast during embryonic stem cell differentiation. Development, 130(17):4217-4227, September 2003. URL http://eutils.ncbi.nlm.nih.gov/entrez/eutils/elink.fcgi?dbfrom=pubmed\&id=12874139\& retmode=ref\&cmd=prlinks.

[17] Costis Papanayotou, Ataaillah Benhaddou, Anne Camus, Aitana Perea-Gomez, Alice Jouneau, Valérie Mezger, Francina Langa, Sascha Ott, Délara Sabéran-Djoneidi, and Jérôme Collignon. A novel nodal enhancer dependent on pluripotency factors and $\operatorname{smad} 2 / 3$ signaling conditions a regulatory switch during epiblast maturation. Plos Biol, 12(6):e1001890, June 2014. doi: 10.1371/journal.pbio.1001890. URL http://eutils.ncbi.nlm.nih.gov/entrez/eutils/elink.fcgi?dbfrom=pubmed\&id=24960041\& retmode=ref\&cmd=prlinks.

[18] Palle Serup, Carsten Gustavsen, Tino Klein, Leah A Potter, Robert Lin, Nandita Mullapudi, Ewa Wandzioch, Angela Hines, Ashley Davis, Christine Bruun, Nina Engberg, Dorthe R Petersen, Janny M L Peterslund, Raymond J MacDonald, Anne Grapin-Botton, Mark A Magnuson, and Kenneth S Zaret. Partial promoter substitutions generating transcriptional sentinels of diverse signaling pathways in embryonic stem cells and mice. Dis Model Mech, 5(6):956-966, November 2012. doi: 10.1242/dmm.009696. URL http://eutils.ncbi.nlm.nih.gov/entrez/eutils/elink.fcgi?dbfrom= pubmed\&id=22888097\&retmode=ref\&cmd=prlinks.

[19] Fernando Faunes, Penelope Hayward, Silvia Muñoz Descalzo, Sujash S Chatterjee, Tina Balayo, Jamie Trott, Andrew Christoforou, Anna Ferrer-Vaquer, Anna-Katerina Hadjantonakis, Ramanuj Dasgupta, and Alfonso Martinez Arias. A membrane-associated $\beta$-catenin/Oct4 complex correlates with groundstate pluripotency in mouse embryonic stem cells. Development, 140(6):1171-1183, March 2013. doi: 10.1242/dev.085654. URL http://eutils.ncbi.nlm.nih.gov/entrez/eutils/elink.fcgi?dbfrom= pubmed\&id=23444350\&retmode=ref\&cmd=prlinks.

[20] Anna Ferrer-Vaquer, Anna Piliszek, Guangnan Tian, Robert J Aho, Daniel Dufort, and Anna-Katerina Hadjantonakis. A sensitive and bright single-cell resolution live imaging reporter of Wnt/B-catenin signaling in the mouse. BMC Dev Biol, 10(1):121, 2010. doi: 10.1186/1471-213X-10-121. URL http://www . biomedcentral. com/1471-213X/10/121.

[21] Tibor Kalmar, Chea Lim, Penelope Hayward, Silvia Muñoz-Descalzo, Jennifer Nichols, Jordi GarciaOjalvo, and Alfonso Martinez Arias. Regulated Fluctuations in Nanog Expression Mediate Cell Fate Decisions in Embryonic Stem Cells. Plos Biol, 7(7):e1000149, July 2009. doi: 10.1371/journal.pbio. 1000149.g008. URL http://dx.plos.org/10.1371/journal .pbio.1000149.g008.

[22] David A Turner, Jamie Trott, Penelope Hayward, Pau Rué, and Alfonso Martinez Arias. An interplay between extracellular signalling and the dynamics of the exit from pluripotency drives cell fate decisions in mouse ES cells. Biology Open, 3(7):614-626, July 2014. doi: 10.1242/bio.20148409. URL http: //bio.biologists.org/content/early/2014/06/13/bio.20148409.full.pdf+html.

[23] David A Turner, Pau Rué, Jonathan P Mackenzie, Eleanor Davies, and Alfonso Martinez Arias. Brachyury cooperates with Wnt/ $\beta$-Catenin signalling to elicit Primitive Streak like behaviour in differentiating mouse ES cells. BMC Biology, 12(1):63, August 2014. doi: 10.1186/s12915-014-0063-7. URL http://eutils.ncbi.nlm.nih.gov/entrez/eutils/elink.fcgi?dbfrom=pubmed\&id=25115237\& retmode=ref\&cmd=prlinks.

[24] William C Skarnes, Barry Rosen, Anthony P West, Manousos Koutsourakis, Wendy Bushell, Vivek Iyer, Alejandro O Mujica, Mark Thomas, Jennifer Harrow, Tony Cox, David Jackson, Jessica Severin, Patrick Biggs, Jun Fu, Michael Nefedov, Pieter J de Jong, A Francis Stewart, and Allan Bradley. A conditional knockout resource for the genome-wide study of mouse gene function. Nature, 474(7351):337-342, June 2011. doi: 10.1038/nature10163. URL http://eutils.ncbi.nlm.nih.gov/entrez/eutils/elink. fcgi?dbfrom=pubmed\&id=21677750\&retmode=ref\&cmd=prlinks.

[25] Christian Schröter, Pau Rué, Jonathan Peter Mackenzie, and Alfonso Martinez Arias. FGF/MAPK signaling sets the switching threshold of a bistable circuit controlling cell fate decisions in embryonic stem cells. 
bioRxiv preprint doi: https://doi.org/10.1101/051722; this version posted May 13, 2016. The copyright holder for this preprint (which was not certified by peer review) is the author/funder, who has granted bioRxiv a license to display the preprint in perpetuity. It is made available under aCC-BY 4.0 International license.

Development, 142(24):4205-4216, December 2015. doi: 10.1242/dev.127530. URL http://eutils.ncbi. nlm.nih.gov/entrez/eutils/elink.fcgi?dbfrom=pubmed\&id=26511924\&retmode=ref\&cmd=prlinks.

[26] Johannes Schindelin, Ignacio Arganda-Carreras, Erwin Frise, Verena Kaynig, Mark Longair, Tobias Pietzsch, Stephan Preibisch, Curtis Rueden, Stephan Saalfeld, and Benjamin Schmid. Fiji: an opensource platform for biological-image analysis. Nature Methods, 9(7):676-682, 2012. doi: doi:10.1038/ nmeth.2019. URL http://www. nature.com/nmeth/journal/v9/n7/full/nmeth.2019.html\%3FWT.ec_ id\%3DNMETH-201207.

[27] Daniele Soroldoni, David J Jörg, Luis G Morelli, David L Richmond, Johannes Schindelin, Frank Jülicher, and Andrew C Oates. Genetic oscillations. A Doppler effect in embryonic pattern formation. Science, 345(6193):222-225, July 2014. doi: 10.1126/science.1253089. URL http://eutils.ncbi.nlm.nih.gov/ entrez/eutils/elink.fcgi?dbfrom=pubmed\&id=25013078\&retmode=ref\&cmd=prlinks.

[28] David G Wilkinson, Sangita Bhatt, and Bernhard G Herrmann. Expression pattern of the mouse T gene and its role in mesoderm formation. Nature, 343(6259):657-659, February 1990. doi: 10.1038/343657a0. URL http://www. nature.com/doifinder/10.1038/343657a0.

[29] B G Herrmann. Expression pattern of the Brachyury gene in whole-mount TWis/TWis mutant embryos. Development, 113(3):913-917, November 1991. URL http://eutils.ncbi.nlm.nih.gov/entrez/ eutils/elink.fcgi?dbfrom=pubmed\&id=1821859\&retmode=ref\&cmd=prlinks.

[30] R S Beddington, P Rashbass, and V Wilson. Brachyury - a gene affecting mouse gastrulation and early organogenesis. Dev. Suppl., pages 157-165, 1992. URL http://eutils.ncbi.nlm.nih.gov/entrez/ eutils/elink. fcgi?dbfrom=pubmed\&id=1299362\&retmode=ref\&cmd=prlinks.

[31] P P Tam and R R Behringer. Mouse gastrulation: the formation of a mammalian body plan. Mech. Dev., 68(1-2):3-25, November 1997. URL http://eutils.ncbi.nlm.nih.gov/entrez/eutils/elink.fcgi? dbfrom=pubmed\&id=9431800\&retmode=ref\&cmd=prlinks.

[32] Patrick P L Tam and David A F Loebel. Gene function in mouse embryogenesis: get set for gastrulation. Nat Rev Genet, 8(5):368-381, March 2007. doi: 10.1038/nrg2084. URL http://www .nature.com/ doifinder/10.1038/nrg2084.

[33] X Zhou, H Sasaki, L Lowe, B L Hogan, and M R Kuehn. Nodal is a novel TGF-beta-like gene expressed in the mouse node during gastrulation. Nature, 361(6412):543-547, February 1993. doi: 10.1038/ 361543a0. URL http://eutils.ncbi.nlm.nih.gov/entrez/eutils/elink.fcgi?dbfrom=pubmed\&id= 8429908\&retmode=ref\&cmd=prlinks.

[34] F L Conlon, K M Lyons, N Takaesu, K S Barth, A Kispert, B Herrmann, and E J Robertson. A primary requirement for nodal in the formation and maintenance of the primitive streak in the mouse. Development, 120(7):1919-1928, July 1994. URL http://eutils.ncbi.nlm.nih.gov/entrez/eutils/ elink. fcgi?dbfrom=pubmed\&id=7924997\&retmode=ref\&cmd=prlinks.

[35] Baozhi Chen, Michael E Dodge, Wei Tang, Jianming Lu, Zhiqiang Ma, Chih-Wei Fan, Shuguang Wei, Wayne Hao, Jessica Kilgore, Noelle S Williams, Michael G Roth, James F Amatruda, Chuo Chen, and Lawrence Lum. Small molecule-mediated disruption of Wnt-dependent signaling in tissue regeneration and cancer. Nat Chem Biol, 5(2):100-107, January 2009. doi: 10.1038/nchembio.137. URL http: //www.nature.com/doifinder/10.1038/nchembio.137.

[36] Shih-Min A Huang, Yuji M Mishina, Shanming Liu, Atwood Cheung, Frank Stegmeier, Gregory A Michaud, Olga Charlat, Elizabeth Wiellette, Yue Zhang, Stephanie Wiessner, Marc Hild, Xiaoying Shi, Christopher J Wilson, Craig Mickanin, Vic Myer, Aleem Fazal, Ronald Tomlinson, Fabrizio Serluca, Wenlin Shao, Hong Cheng, Michael Shultz, Christina Rau, Markus Schirle, Judith Schlegl, Sonja Ghidelli, Stephen Fawell, Chris Lu, Daniel Curtis, Marc W Kirschner, Christoph Lengauer, Peter M Finan, John A Tallarico, Tewis Bouwmeester, Jeffery A Porter, Andreas Bauer, and Feng Cong. Tankyrase inhibition stabilizes axin and antagonizes Wnt signalling. Nature, 461(7264):614-620, October 2009. doi: 10.1038/nature08356. URL http://eutils.ncbi.nlm.nih.gov/entrez/eutils/elink.fcgi?dbfrom= pubmed\&id=19759537\&retmode $=$ ref $\& \mathrm{cmd}=$ prlinks.

[37] David A Turner, Peter Baillie-Johnson, and Alfonso Martinez Arias. Organoids and the genetically encoded self-assembly of embryonic stem cells. Bioessays, 38(2):181-191, February 2016. doi: 10.1002/bies. 201500111. URL http://eutils.ncbi.nlm.nih.gov/entrez/eutils/elink.fcgi?dbfrom=pubmed\&id= 26666846\&retmode=ref\&cmd=prlinks. 
bioRxiv preprint doi: https://doi.org/10.1101/051722; this version posted May 13, 2016. The copyright holder for this preprint (which was not certified by peer review) is the author/funder, who has granted bioRxiv a license to display the preprint in perpetuity. It is made available under aCC-BY 4.0 International license.

[38] Nadav Ben-Haim, Cindy Lu, Marcela Guzman-Ayala, Luca Pescatore, Daniel Mesnard, Mirko Bischofberger, Felix Naef, Elizabeth J Robertson, and Daniel B Constam. The nodal precursor acting via activin receptors induces mesoderm by maintaining a source of its convertases and BMP4. Developmental Cell, 11(3):313-323, September 2006. doi: 10.1016/j.devcel.2006.07.005. URL http://eutils.ncbi.nlm.nih. gov/entrez/eutils/elink. fcgi?dbfrom=pubmed\&id=16950123\&retmode=ref\&cmd=prlinks.

[39] G Winnier, M Blessing, P A Labosky, and B L Hogan. Bone morphogenetic protein-4 is required for mesoderm formation and patterning in the mouse. Genes $\&$ Development, 9(17):2105-2116, September 1995. URL http://eutils.ncbi.nlm.nih.gov/entrez/eutils/elink.fcgi?dbfrom=pubmed\&id= 7657163\&retmode=ref\&cmd=prlinks.

[40] Mattias Hansson, Dorthe R Olesen, Janny M L Peterslund, Nina Engberg, Morten Kahn, Maria Winzi, Tino Klein, Poul Maddox-Hyttel, and Palle Serup. A late requirement for Wnt and FGF signaling during activin-induced formation of foregut endoderm from mouse embryonic stem cells. Developmental Biology, 330(2):286-304, June 2009. doi: 10.1016/j.ydbio.2009.03.026. URL http://eutils.ncbi.nlm.nih.gov/ entrez/eutils/elink. f cgi?dbfrom=pubmed\&id=19358838\&retmode=ref\&cmd=prlinks.

[41] Steven A Jackson, Jacqueline Schiesser, Edouard G Stanley, and Andrew G Elefanty. Differentiating Embryonic Stem Cells Pass through 'Temporal Windows' That Mark Responsiveness to Exogenous and Paracrine Mesendoderm Inducing Signals. PLoS ONE, 5(5):e10706, May 2010. doi: 10.1371/journal.pone. 0010706.g007. URL http://dx.plos.org/10.1371/journal .pone.0010706.g007.

[42] J Brennan, C C Lu, D P Norris, T A Rodriguez, R S Beddington, and E J Robertson. Nodal signalling in the epiblast patterns the early mouse embryo. Nature, 411(6840):965-969, June 2001. doi: 10.1038/ 35082103. URL http://eutils.ncbi.nlm.nih.gov/entrez/eutils/elink.fcgi?dbfrom=pubmed\&id= 11418863\&retmode=ref\&cmd=prlinks.

[43] Cindy C Lu and Elizabeth J Robertson. Multiple roles for Nodal in the epiblast of the mouse embryo in the establishment of anterior-posterior patterning. Developmental Biology, 273(1):149-159, September 2004. doi: 10.1016/j.ydbio.2004.06.004. URL http://eutils.ncbi.nlm.nih.gov/entrez/eutils/ elink.fcgi?dbfrom=pubmed\&id=15302604\&retmode=ref\&cmd=prlinks.

[44] I Varlet, J Collignon, and E J Robertson. nodal expression in the primitive endoderm is required for specification of the anterior axis during mouse gastrulation. Development, 124(5):1033-1044, March 1997. URL http://eutils.ncbi.nlm.nih.gov/entrez/eutils/elink.fcgi?dbfrom=pubmed\& id=9056778\&retmode=ref\&cmd=prlinks.

[45] L A Lowe, D M Supp, K Sampath, T Yokoyama, C V Wright, S S Potter, P Overbeek, and M R Kuehn. Conserved left-right asymmetry of nodal expression and alterations in murine situs inversus. Nature, 381 (6578):158-161, May 1996. doi: 10.1038/381158a0. URL http://eutils.ncbi.nlm.nih.gov/entrez/ eutils/elink. f cgi?dbfrom=pubmed\&id=8610013\&retmode=ref \&cmd=prlinks.

[46] J Collignon, I Varlet, and E J Robertson. Relationship between asymmetric nodal expression and the direction of embryonic turning. Nature, 381(6578):155-158, May 1996. doi: 10.1038/ 381155a0. URL http://eutils.ncbi.nlm.nih.gov/entrez/eutils/elink.fcgi?dbfrom=pubmed\&id= 8610012\&retmode=ref\&cmd=prlinks.

[47] C Meno, A Shimono, Y Saijoh, K Yashiro, K Mochida, S Ohishi, S Noji, H Kondoh, and H Hamada. lefty-1 is required for left-right determination as a regulator of lefty-2 and nodal. Cell, 94(3):287-297, August 1998. URL http://eutils.ncbi.nlm.nih.gov/entrez/eutils/elink.fcgi?dbfrom=pubmed\& id=9708731\&retmode $=$ ref\&cmd=prlinks.

[48] Katsuyoshi Takaoka, Masamichi Yamamoto, Hidetaka Shiratori, Chikara Meno, Janet Rossant, Yukio Saijoh, and Hiroshi Hamada. The mouse embryo autonomously acquires anterior-posterior polarity at implantation. Developmental Cell, 10(4):451-459, April 2006. doi: 10.1016/j.devcel.2006.02.017. URL http://eutils.ncbi.nlm.nih.gov/entrez/eutils/elink.fcgi?dbfrom=pubmed\&id=16580991\& retmode=ref\&cmd=prlinks.

[49] Aitana Perea-Gomez, Francis D J Vella, William Shawlot, Mustapha Oulad-Abdelghani, Claire Chazaud, Chikara Meno, Veronique Pfister, Lan Chen, Elizabeth Robertson, Hiroshi Hamada, Richard R Behringer, and Siew-Lan Ang. Nodal antagonists in the anterior visceral endoderm prevent the formation of multiple primitive streaks. Developmental Cell, 3(5):745-756, November 2002. URL http://eutils.ncbi.nlm. nih.gov/entrez/eutils/elink.fcgi?dbfrom=pubmed\&id=12431380\&retmode=ref\&cmd=prlinks. 
bioRxiv preprint doi: https://doi.org/10.1101/051722; this version posted May 13, 2016. The copyright holder for this preprint (which was not certified by peer review) is the author/funder, who has granted bioRxiv a license to display the preprint in perpetuity. It is made available under aCC-BY 4.0 International license.

[50] Masamichi Yamamoto, Yukio Saijoh, Aitana Perea-Gomez, William Shawlot, Richard R Behringer, SiewLan Ang, Hiroshi Hamada, and Chikara Meno. Nodal antagonists regulate formation of the anteroposterior axis of the mouse embryo. Nature, 428(6981):387-392, March 2004. doi: 10.1038/nature02418. URL http://eutils.ncbi.nlm.nih.gov/entrez/eutils/elink.fcgi?dbfrom=pubmed\&id=15004567\& retmode=ref\&cmd=prlinks.

[51] Samara L Lewis, Poh-Lynn Khoo, R Andrea De Young, Kirsten Steiner, Chris Wilcock, Mahua Mukhopadhyay, Heiner Westphal, Robyn V Jamieson, Lorraine Robb, and Patrick P L Tam. Dkk1 and Wnt3 interact to control head morphogenesis in the mouse. Development, 135(10):1791-1801, May 2008. doi: 10.1242/dev.018853. URL http://eutils.ncbi.nlm.nih.gov/entrez/eutils/elink.fcgi?dbfrom= pubmed\&id=18403408\&retmode=ref\&cmd=prlinks.

[52] Kimberly E Inman and Karen M Downs. Localization of Brachyury (T) in embryonic and extraembryonic tissues during mouse gastrulation. Gene Expr. Patterns, 6(8):783-793, October 2006. doi: 10.1016/ j.modgep.2006.01.010. URL http://eutils.ncbi.nlm.nih.gov/entrez/eutils/elink.fcgi?dbfrom= pubmed\&id=16545989\&retmode=ref\&cmd=prlinks.

[53] Karen M Downs, Kimberly E Inman, Dexter X Jin, and Allen C Enders. The Allantoic Core Domain: new insights into development of the murine allantois and its relation to the primitive streak. Dev. Dyn., 238 (3):532-553, March 2009. doi: 10.1002/dvdy.21862. URL http://eutils.ncbi.nlm.nih.gov/entrez/ eutils/elink. fcgi?dbfrom=pubmed\&id=19191225\&retmode=ref\&cmd=prlinks.

[54] Helen K Chea, Christopher V Wright, and Billie J Swalla. Nodal signaling and the evolution of deuterostome gastrulation. Dev. Dyn., 234(2):269-278, October 2005. doi: 10.1002/dvdy.20549. URL http://eutils.ncbi.nlm.nih.gov/entrez/eutils/elink.fcgi?dbfrom=pubmed\&id=16127715\& retmode $=$ ref\&cmd=prlinks.

[55] Michael M Shen. Nodal signaling: developmental roles and regulation. Development, 134(6):1023-1034, March 2007. doi: 10.1242/dev.000166. URL http://eutils.ncbi.nlm.nih.gov/entrez/eutils/elink. fcgi?dbfrom=pubmed\&id=17287255\&retmode=ref\&cmd=prlinks.

[56] Patrick Müller, Katherine W Rogers, Ben M Jordan, Joon S Lee, Drew Robson, Sharad Ramanathan, and Alexander F Schier. Differential diffusivity of Nodal and Lefty underlies a reaction-diffusion patterning system. Science, 336(6082):721-724, May 2012. doi: 10.1126/science.1221920. URL http://eutils.ncbi. nlm.nih.gov/entrez/eutils/elink.fcgi?dbfrom=pubmed\&id=22499809\&retmode=ref\&cmd=prlinks.

[57] Tetsuya Nakamura, Naoki Mine, Etsushi Nakaguchi, Atsushi Mochizuki, Masamichi Yamamoto, Kenta Yashiro, Chikara Meno, and Hiroshi Hamada. Generation of robust left-right asymmetry in the mouse embryo requires a self-enhancement and lateral-inhibition system. Developmental Cell, 11(4):495-504, October 2006. doi: 10.1016/j.devcel.2006.08.002. URL http://eutils.ncbi.nlm.nih.gov/entrez/eutils/ elink. f cgi ?dbfrom=pubmed\&id=17011489\&retmode=ref\&cmd=prlinks.

[58] Hou Juan and Hiroshi Hamada. Roles of nodal-lefty regulatory loops in embryonic patterning of vertebrates. Genes to Cells, 6(11):923-930, November 2001. doi: 10.1046/j.1365-2443.2001.00481.x. URL http://doi.wiley.com/10.1046/j.1365-2443.2001.00481.x.

[59] Katherine E Galvin-Burgess, Emily D Travis, Kelsey E Pierson, and Jay L Vivian. TGF- $\beta$-superfamily signaling regulates embryonic stem cell heterogeneity: self-renewal as a dynamic and regulated equilibrium. Stem Cells, 31(1):48-58, January 2013. doi: 10.1002/stem.1252. URL http://eutils.ncbi.nlm.nih. gov/entrez/eutils/elink. fcgi?dbfrom=pubmed\&id=23081664\&retmode=ref\&cmd=prlinks.

[60] Séverine Beck, J Ann Le Good, Marcela Guzman, Nadav Ben Haim, Karine Roy, Friedrich Beermann, and Daniel B Constam. Extraembryonic proteases regulate Nodal signalling during gastrulation. Nature Cell Biology, 4(12):981-985, November 2002. doi: 10.1038/ncb890. URL http://www.nature.com/ doifinder/10.1038/ncb890.

[61] Hiroshi Hamada. In search of Turing in vivo: understanding Nodal and Lefty behavior. Developmental Cell, 22(5):911-912, May 2012. doi: 10.1016/j.devcel.2012.05.003. URL http://eutils.ncbi.nlm.nih. gov/entrez/eutils/elink.f cgi?dbfrom=pubmed\&id=22595667\&retmode=ref\&cmd=prlinks.

[62] Richard Baldock, Jonathan Bard, Duncan Davidson, and Kirstie Lawson. Theiler Stage Definition, May 1998. URL http://www.emouseatlas.org/emap/ema/theiler_stages/StageDefinition/ stagedefinition.html. 
bioRxiv preprint doi: https://doi.org/10.1101/051722; this version posted May 13, 2016. The copyright holder for this preprint (which was not certified by peer review) is the author/funder, who has granted bioRxiv a license to display the preprint in perpetuity. It is made available under aCC-BY 4.0 International license.

[63] H. Steinbeisser, E.M. De Robertis, M Ku, D S Kessler, and D A Melton. Xenopus axis formation: induction of goosecoid by injected Xwnt-8 and activin mRNAs. Development, 118(2):499-507, June 1993. URL http://eutils.ncbi.nlm.nih.gov/entrez/eutils/elink.fcgi?dbfrom=pubmed\&id= 7900991\&retmode=ref\&cmd=prlinks.

[64] T Watabe, S Kim, A Candia, U Rothbächer, C Hashimoto, K Inoue, and K W Cho. Molecular mechanisms of Spemann's organizer formation: conserved growth factor synergy between Xenopus and mouse. 1995. URL http://genesdev. cshlp.org/content/9/24/3038. short.

[65] D J Crease, S Dyson, and J B Gurdon. Cooperation between the activin and Wnt pathways in the spatial control of organizer gene expression. Proceedings of the National Academy of Sciences, 95(8):4398-4403, April 1998. URL http://eutils.ncbi.nlm.nih.gov/entrez/eutils/elink.fcgi?dbfrom=pubmed\&id= 9539748\&retmode=ref\&cmd=prlinks.

[66] K Joubin and C D Stern. Molecular Interactions Continuously Define the Organizer during the Cell Movements of Gastrulation. Cell, 1999. URL http://www.sciencedirect.com/science/article/pii/ s0092867400800446.

[67] I Skromne and C D Stern. Interactions between Wnt and Vg1 signalling pathways initiate primitive streak formation in the chick embryo. Development, 128(15):2915-2927, August 2001. URL http://eutils.ncbi.nlm.nih.gov/entrez/eutils/elink.fcgi?dbfrom=pubmed\&id=11532915\& retmode=ref\&cmd=prlinks.

[68] Bradley J Merrill, H Amalia Pasolli, Lisa Polak, Michael Rendl, Maria J García-García, Kathryn V Anderson, and Elaine Fuchs. Tcf3: a transcriptional regulator of axis induction in the early embryo. Development, 131(2):263-274, January 2004. doi: 10.1242/dev.00935. URL http://eutils.ncbi.nlm. nih.gov/entrez/eutils/elink.fcgi?dbfrom=pubmed\&id=14668413\&retmode=ref\&cmd=prlinks.

[69] H Pöpperl, C Schmidt, V Wilson, C R Hume, J Dodd, R Krumlauf, and R S Beddington. Misexpression of Cwnt8C in the mouse induces an ectopic embryonic axis and causes a truncation of the anterior neuroectoderm. Development, 124(15):2997-3005, August 1997. URL http://eutils.ncbi.nlm.nih. gov/entrez/eutils/elink.fcgi?dbfrom=pubmed\&id=9247341\&retmode=ref\&cmd=prlinks.

[70] William C Dunty, Kristin K Biris, Ravindra B Chalamalasetty, Makoto M Taketo, Mark Lewandoski, and Terry $\mathrm{P}$ Yamaguchi. Wnt3a/beta-catenin signaling controls posterior body development by coordinating mesoderm formation and segmentation. Development, 135(1):85-94, January 2008. doi: 10.1242/dev.009266. URL http://eutils.ncbi.nlm.nih.gov/entrez/eutils/elink.fcgi?dbfrom= pubmed\&id=18045842\&retmode=ref\&cmd=prlinks.

[71] J Sampedro, P Johnston, and P A Lawrence. A role for wingless in the segmental gradient of Drosophila? Development, 117(2):677-687, February 1993. URL http://eutils.ncbi.nlm.nih.gov/ entrez/eutils/elink. fcgi?dbfrom=pubmed\&id=8330533\&retmode=ref\&cmd=prlinks.

[72] M K Baylies, Alfonso Martinez Arias, and M Bate. wingless is required for the formation of a subset of muscle founder cells during Drosophila embryogenesis. Development, 121(11):3829-3837, November 1995. URL http://eutils.ncbi.nlm.nih.gov/entrez/eutils/elink.fcgi?dbfrom=pubmed\&id= 8582292\&retmode=ref\&cmd=prlinks.

[73] M J García-García, P Ramain, P Simpson, and J Modolell. Different contributions of pannier and wingless to the patterning of the dorsal mesothorax of Drosophila. Development, 126(16):3523-3532, August 1999. URL http://eutils.ncbi.nlm.nih.gov/entrez/eutils/elink.fcgi?dbfrom=pubmed\& id=10409499\&retmode=ref\&cmd=prlinks.

[74] C P Heisenberg, M Tada, G J Rauch, L Saude, M L Concha, R Geisler, D L Stemple, J C Smith, and S W Wilson. Silberblick/Wnt11 mediates convergent extension movements during zebrafish gastrulation. Nature, 405(6782):76-81, May 2000. doi: 10.1038/35011068. URL http://eutils.ncbi.nlm.nih.gov/ entrez/eutils/elink.fcgi?dbfrom=pubmed\&id=10811221\&retmode=ref\&cmd=prlinks.

[75] Ana M Mateus, Nicole Gorfinkiel, and Alfonso Martinez Arias. Origin and function of fluctuations in cell behaviour and the emergence of patterns. Semin Cell Dev Biol, 20(7):877-884, September 2009. doi: 10.1016/j.semcdb.2009.07.009. URL http://linkinghub.elsevier.com/retrieve/pii/ S1084952109001566.

[76] Alfonso Martinez Arias and Penelope Hayward. Filtering transcriptional noise during development: concepts and mechanisms. Nat Rev Genet, 7(1):34-44, January 2006. doi: 10.1038/nrg1750. URL http://www . nature.com/doifinder/10.1038/nrg1750. 
bioRxiv preprint doi: https://doi.org/10.1101/051722; this version posted May 13, 2016. The copyright holder for this preprint (which was not certified by peer review) is the author/funder, who has granted bioRxiv a license to display the preprint in perpetuity. It is made available under aCC-BY 4.0 International license.

[77] A Bejsovec and Alfonso Martinez Arias. Roles of wingless in patterning the larval epidermis of Drosophila. Development, 113(2):471-485, October 1991. URL http://eutils.ncbi.nlm.nih.gov/entrez/eutils/ elink.fcgi?dbfrom=pubmed\&id=1782860\&retmode=ref\&cmd=prlinks.

[78] Cyrille Alexandre, Alberto Baena-Lopez, and Jean-Paul Vincent. Patterning and growth control by membrane-tethered Wingless. Nature, 505(7482):180-185, January 2014. doi: 10.1038/nature12879. URL http://eutils.ncbi.nlm.nih.gov/entrez/eutils/elink.fcgi?dbfrom=pubmed\&id=24390349\& retmode=ref\&cmd=prlinks.

[79] J P Couso, S A Bishop, and Alfonso Martinez Arias. The wingless signalling pathway and the patterning of the wing margin in Drosophila. Development, 120(3):621-636, March 1994. URL http://eutils.ncbi . nlm.nih.gov/entrez/eutils/elink.fcgi?dbfrom=pubmed\&id=8162860\&retmode=ref\&cmd=prlinks.

[80] Silvia Muñoz-Descalzo, Joaquin de Navascues, and Alfonso Martinez Arias. Wnt-Notch signalling: an integrated mechanism regulating transitions between cell states. Bioessays, 34(2):110-118, February 2012. doi: 10.1002/bies.201100102. URL http://eutils.ncbi.nlm.nih.gov/entrez/eutils/elink.fcgi? dbfrom=pubmed\&id=22215536\&retmode=ref\&cmd=prlinks.

[81] G G Tortelote and J Manuel Hernández-Hernández. Wnt3 function in the epiblast is required for the maintenance but not the initiation of gastrulation in mice. Developmental Biology, 2012. URL http: //www.sciencedirect.com/science/article/pii/S0012160612005544.

[82] Jaime A Rivera-Pérez and Terry Magnuson. Primitive streak formation in mice is preceded by localized activation of Brachyury and Wnt3. Developmental Biology, 288(2):363-371, December 2005. doi: 10.1016/ j.ydbio.2005.09.012. URL http://linkinghub.elsevier.com/retrieve/pii/S0012160605006196.

[83] T A Pelton, S Sharma, T C Schulz, J Rathjen, and P D Rathjen. Transient pluripotent cell populations during primitive ectoderm formation: correlation of in vivo and in vitro pluripotent cell development. $J$. Cell. Sci., 115(Pt 2):329-339, January 2002. URL http://eutils.ncbi.nlm.nih.gov/entrez/eutils/ elink. f cgi ?dbfrom=pubmed\&id=11839785\&retmode=ref\&cmd=prlinks.

[84] D A Rappolee, C Basilico, Y Patel, and Z Werb. Expression and function of FGF-4 in peri-implantation development in mouse embryos. Development, 120(8):2259-2269, August 1994. URL http://eutils.ncbi. nlm.nih.gov/entrez/eutils/elink.fcgi?dbfrom=pubmed\&id=7925026\&retmode=ref\&cmd=prlinks.

[85] Jackson A Hoffman, Chun-I Wu, and Bradley J Merrill. Tcf7l1 prepares epiblast cells in the gastrulating mouse embryo for lineage specification. Development, 140(8):1665-1675, April 2013. doi: 10.1242/dev.087387. URL http://eutils.ncbi.nlm.nih.gov/entrez/eutils/elink.fcgi?dbfrom= pubmed\&id=23487311\&retmode=ref\&cmd=prlinks.

[86] Anne Camus, Aitana Perea-Gomez, Anne Moreau, and Jérôme Collignon. Absence of Nodal signaling promotes precocious neural differentiation in the mouse embryo. Developmental Biology, 295(2):743-755, July 2006. doi: 10.1016/j.ydbio.2006.03.047. URL http://linkinghub.elsevier.com/retrieve/pii/ S0012160606002582.

[87] Steffen Biechele, Brian J Cox, and Janet Rossant. Porcupine homolog is required for canonical Wnt signaling and gastrulation in mouse embryos. Developmental Biology, 355(2):275-285, July 2011. doi: 10.1016/ j.ydbio.2011.04.029. URL http://linkinghub.elsevier.com/retrieve/pii/S0012160611002764.

[88] S Biechele, K Cockburn, F Lanner, and B J Cox. Porcn-dependent Wnt signaling is not required prior to mouse gastrulation. Development, 140(13):2961-2971, 2013. doi: 10.1242/dev.094458. URL http: //dev.biologists.org/content/early/2013/06/12/dev.094458. short.

[89] Caroline Kemp, Erik Willems, Shaaban Abdo, Louis Lambiv, and Luc Leyns. Expression of all Wnt genes and their secreted antagonists during mouse blastocyst and postimplantation development. Dev. Dyn., 233(3):1064-1075, July 2005. doi: 10.1002/dvdy.20408. URL http://eutils.ncbi.nlm.nih.gov/ entrez/eutils/elink. fcgi?dbfrom=pubmed\&id=15880404\&retmode=ref\&cmd=prlinks.

[90] M P Stavridis, J S Lunn, B J Collins, and K G Storey. A discrete period of FGF-induced Erk1/2 signalling is required for vertebrate neural specification. Development, 134(16):2889-2894, July 2007. doi: 10.1242/dev.02858. URL http://dev.biologists.org/cgi/doi/10.1242/dev.02858.

[91] T Kunath, M K Saba-El-Leil, M Almousailleakh, J Wray, S Meloche, and A Smith. FGF stimulation of the Erk1/2 signalling cascade triggers transition of pluripotent embryonic stem cells from self-renewal to lineage commitment. Development, 134(16):2895-2902, July 2007. doi: 10.1242/dev.02880. URL http://dev.biologists.org/cgi/doi/10.1242/dev.02880. 
bioRxiv preprint doi: https://doi.org/10.1101/051722; this version posted May 13, 2016. The copyright holder for this preprint (which was not certified by peer review) is the author/funder, who has granted bioRxiv a license to display the preprint in perpetuity. It is made available under aCC-BY 4.0 International license.

[92] Boris Greber, Guangming Wu, Christof Bernemann, Jin Young Joo, Dong Wook Han, Kinarm Ko, Natalia Tapia, Davood Sabour, Jared Sterneckert, Paul Tesar, and Hans R Schöler. Conserved and divergent roles of FGF signaling in mouse epiblast stem cells and human embryonic stem cells. Cell Stem Cell, 6(3):215226, March 2010. doi: 10.1016/j.stem.2010.01.003. URL http://eutils.ncbi.nlm.nih.gov/entrez/ eutils/elink. fcgi?dbfrom=pubmed\&id=20207225\&retmode=ref\&cmd=prlinks.

[93] Jared Sterneckert, Martin Stehling, Christof Bernemann, Marcos J Araúzo-Bravo, Boris Greber, Luca Gentile, Claudia Ortmeier, Martina Sinn, Guangming Wu, David Ruau, Martin Zenke, Rhea Brintrup, Diana C Klein, Kinarm Ko, and Hans R Schöler. Neural Induction Intermediates Exhibit Distinct Roles of Fgf Signaling. Stem Cells, 28(10):1772-1781, October 2010. doi: 10.1002/stem.498. URL http: //doi.wiley.com/10.1002/stem.498.

[94] Anne M Boulet and Mario R Capecchi. Signaling by FGF4 and FGF8 is required for axial elongation of the mouse embryo. Developmental Biology, 371(2):235-245, November 2012. doi: 10.1016/j.ydbio.2012.08.017. URL http://linkinghub.elsevier.com/retrieve/pii/S0012160612004575.

[95] B G Ciruna, L Schwartz, K Harpal, T P Yamaguchi, and J Rossant. Chimeric analysis of fibroblast growth factor receptor-1 (Fgfr1) function: a role for FGFR1 in morphogenetic movement through the primitive streak. Development, 124(14):2829-2841, July 1997. URL http://dev.biologists.org/content/124/ 14/2829. abstract.

[96] C X Deng, A Wynshaw-Boris, M M Shen, C Daugherty, D M Ornitz, and P Leder. Murine FGFR-1 is required for early postimplantation growth and axial organization. Genes \& Development, 8(24):30453057, December 1994. doi: 10.1101/gad.8.24.3045. URL http://www.genesdev.org/cgi/doi/10.1101/ $\operatorname{gad} .8 .24 .3045$.

[97] B P Davidson, L Cheng, S J Kinder, and PPL Tam. Exogenous FGF-4 Can Suppress Anterior Development in the Mouse Embryo during Neurulation and Early Organogenesis. Developmental Biology, 2000. URL http://www.sciencedirect.com/science/article/pii/S0012160600996635.

[98] Xiaoxiao Zhang, Kevin A Peterson, X Shirley Liu, Andrew P McMahon, and Shinsuke Ohba. Gene regulatory networks mediating canonical Wnt signal-directed control of pluripotency and differentiation in embryo stem cells. Stem Cells, 31(12):2667-2679, December 2013. doi: 10.1002/stem.1371. URL http://eutils.ncbi.nlm.nih.gov/entrez/eutils/elink.fcgi?dbfrom=pubmed\&id=23505158\& retmode=ref\&cmd=prlinks.

[99] K Dorey and E Amaya. FGF signalling: diverse roles during early vertebrate embryogenesis. Development, 137(22):3731-3742, October 2010. doi: 10.1242/dev.037689. URL http://dev.biologists.org/cgi/ doi/10.1242/dev.037689.

[100] Cantas Alev, Yuping Wu, Yukiko Nakaya, and Guojun Sheng. Decoupling of amniote gastrulation and streak formation reveals a morphogenetic unity in vertebrate mesoderm induction. Development, May 2013. doi: 10.1242/dev.094318. URL http://eutils.ncbi.nlm.nih.gov/entrez/eutils/elink.fcgi? dbf rom=pubmed\&id=23698348\&retmode=ref\&cmd=prlinks.

[101] Madeline A Lancaster, Magdalena Renner, Carol-Anne Martin, Daniel Wenzel, Louise S Bicknell, Matthew E Hurles, Tessa Homfray, Josef M Penninger, Andrew P Jackson, and Juergen A Knoblich. Cerebral organoids model human brain development and microcephaly. Nature, 501(7467):373-379, September 2013. doi: 10.1038/nature12517. URL http://eutils.ncbi.nlm.nih.gov/entrez/eutils/elink. fcgi?dbfrom=pubmed\&id=23995685\&retmode=ref\&cmd=prlinks.

[102] Tokushige Nakano, Satoshi Ando, Nozomu Takata, Masako Kawada, Keiko Muguruma, Kiyotoshi Sekiguchi, Koichi Saito, Shigenobu Yonemura, Mototsugu Eiraku, and Yoshiki Sasai. Self-formation of optic cups and storable stratified neural retina from human ESCs. Cell Stem Cell, 10(6):771-785, June 2012. doi: 10.1016/j.stem.2012.05.009. URL http://eutils.ncbi.nlm.nih.gov/entrez/eutils/ elink. fcgi?dbfrom=pubmed\&id=22704518\&retmode=ref\&cmd=prlinks.

[103] Mototsugu Eiraku, Nozomu Takata, Hiroki Ishibashi, Masako Kawada, Eriko Sakakura, Satoru Okuda, Kiyotoshi Sekiguchi, Taiji Adachi, and Yoshiki Sasai. Self-organizing optic-cup morphogenesis in three-dimensional culture. Nature, 472(7341):51-56, April 2011. doi: 10.1038/nature09941. URL http://eutils.ncbi.nlm.nih.gov/entrez/eutils/elink.fcgi?dbfrom=pubmed\&id=21475194\& retmode=ref\&cmd=prlinks. 
bioRxiv preprint doi: https://doi.org/10.1101/051722; this version posted May 13, 2016. The copyright holder for this preprint (which was not certified by peer review) is the author/funder, who has granted bioRxiv a license to display the preprint in perpetuity. It is made available under aCC-BY 4.0 International license.

[104] Mototsugu Eiraku, Kiichi Watanabe, Mami Matsuo-Takasaki, Masako Kawada, Shigenobu Yonemura, Michiru Matsumura, Takafumi Wataya, Ayaka Nishiyama, Keiko Muguruma, and Yoshiki Sasai. Self-organized formation of polarized cortical tissues from ESCs and its active manipulation by extrinsic signals. Cell Stem Cell, 3(5):519-532, November 2008. doi: 10.1016/j.stem.2008.09.002. URL http://eutils.ncbi.nlm.nih.gov/entrez/eutils/elink.fcgi?dbfrom=pubmed\&id=18983967\& retmode=ref\&cmd=prlinks.

[105] David Kimelman. Tales of Tails (and Trunks): Forming the Posterior Body in Vertebrate Embryos. Elsevier Inc., 1 edition, 2016. doi: 10.1016/bs.ctdb.2015.12.008. URL http://dx.doi.org/10.1016/bs . ctdb.2015.12.008.

[106] Domingos Henrique, Elsa Abranches, Laure Verrier, and Kate G Storey. Neuromesodermal progenitors and the making of the spinal cord. Development, 142(17):2864-2875, September 2015. doi: 10.1242/dev.119768. URL http://eutils.ncbi.nlm.nih.gov/entrez/eutils/elink.fcgi?dbfrom= pubmed\&id=26329597\&retmode=ref\&cmd=prlinks.

[107] N Fossat, V Jones, P L Khoo, D Bogani, A Hardy, K Steiner, M Mukhopadhyay, H Westphal, P M Nolan, R Arkell, and P P L Tam. Stringent requirement of a proper level of canonical WNT signalling activity for head formation in mouse embryo. Development, 138(4):667-676, January 2011. doi: 10.1242/dev.052803. URL http://dev. biologists.org/cgi/doi/10.1242/dev. 052803.

[108] Nicolas Fossat, Vanessa Jones, Maria J García-García, and Patrick P L Tam. Modulation of WNT signaling activity is key to the formation of the embryonic head. Cell Cycle, 11(1):26-32, January 2012. doi: 10.4161/cc.11.1.18700. URL http://eutils.ncbi.nlm.nih.gov/entrez/eutils/elink.fcgi?dbfrom= pubmed\&id=22157093\&retmode=ref\&cmd=prlinks.

[109] Claudio D Stern and Karen M Downs. The hypoblast (visceral endoderm): an evo-devo perspective. Development, 139(6):1059-1069, March 2012. doi: 10.1242/dev.070730. URL http://eutils.ncbi.nlm. nih.gov/entrez/eutils/elink. fcgi?dbfrom=pubmed\&id=22354839\&retmode=ref\&cmd=prlinks.

[110] A Perea-Gómez, M Rhinn, and S.L. Ang. Role of the anterior visceral endoderm in restricting posterior signals in the mouse embryo. Int. J. Dev. Biol., 45(1):311-320, 2001. URL http://eutils.ncbi.nlm. nih.gov/entrez/eutils/elink.fcgi?dbfrom=pubmed\&id=11291861\&retmode=ref\&cmd=prlinks.

[111] Cynthia Lilian Andoniadou and Juan Pedro Martinez-Barbera. Developmental mechanisms directing early anterior forebrain specification in vertebrates. Cell. Mol. Life Sci., 70(20):3739-3752, October 2013. doi: 10.1007/s00018-013-1269-5. URL http://eutils.ncbi.nlm.nih.gov/entrez/eutils/ elink. fcgi?dbfrom=pubmed\&id=23397132\&retmode=ref\&cmd=prlinks.

[112] A Di-Gregorio, M Sancho, D W Stuckey, L A Crompton, J Godwin, Y Mishina, and T A Rodriguez. BMP signalling inhibits premature neural differentiation in the mouse embryo. Development, 134(18): 3359-3369, August 2007. doi: 10.1242/dev.005967. URL http://dev.biologists.org/cgi/doi/10. $1242 / \mathrm{dev} .005967$.

[113] N Ray Dunn, Stéphane D Vincent, Leif Oxburgh, Elizabeth J Robertson, and Elizabeth K Bikoff. Combinatorial activities of Smad2 and Smad3 regulate mesoderm formation and patterning in the mouse embryo. Development, 131(8):1717-1728, April 2004. doi: 10.1242/dev.01072. URL http://eutils.ncbi.nlm. nih.gov/entrez/eutils/elink.fcgi?dbfrom=pubmed\&id=15084457\&retmode=ref\&cmd=prlinks.

[114] S D Vincent. Cell fate decisions within the mouse organizer are governed by graded Nodal signals. Genes 85 Development, 17(13):1646-1662, June 2003. doi: 10.1101/gad.1100503. URL http://www.genesdev. org/cgi/doi/10.1101/gad.1100503.

[115] Yeh-Chuin Poh, Junwei Chen, Ying Hong, Haiying Yi, Shuang Zhang, Junjian Chen, Douglas C Wu, Lili Wang, Qiong Jia, Rishi Singh, Wenting Yao, Youhua Tan, Arash Tajik, Tetsuya S Tanaka, and Ning Wang. Generation of organized germ layers from a single mouse embryonic stem cell. Nat Commun, 5:4000, 2014. doi: 10.1038/ncomms5000. URL http://eutils.ncbi.nlm.nih.gov/entrez/eutils/ elink. f cgi ?dbfrom=pubmed\&id=24873804\&retmode=ref\&cmd=prlinks.

[116] Aryeh Warmflash, Benoit Sorre, Fred Etoc, Eric D Siggia, and Ali H Brivanlou. A method to recapitulate early embryonic spatial patterning in human embryonic stem cells. Nature Methods, 11(8):847-854, June 2014. doi: 10.1038/nmeth.3016. URL http://www.nature.com/doifinder/10.1038/nmeth.3016.

[117] A Nagy, M Gertsenstein, K Vintersten, and R Behringer. Karyotyping Mouse Cells. Cold Spring Harbor Protocols, 2008(6):pdb.prot4706-pdb.prot4706, May 2008. doi: 10.1101/pdb.prot4706. URL http: //www . cshprotocols.org/cgi/doi/10.1101/pdb.prot4706. 
bioRxiv preprint doi: https://doi.org/10.1101/051722; this version posted May 13, 2016. The copyright holder for this preprint (which was not certified by peer review) is the author/funder, who has granted bioRxiv a license to display the preprint in perpetuity. It is made available under aCC-BY 4.0 International license.

[118] D B Ring, K W Johnson, E J Henriksen, J M Nuss, D Goff, T R Kinnick, S T Ma, J W Reeder, I Samuels, T Slabiak, A S Wagman, M E W Hammond, and S D Harrison. Selective Glycogen Synthase Kinase 3 Inhibitors Potentiate Insulin Activation of Glucose Transport and Utilization In Vitro and In Vivo. Diabetes, 52(3):588-595, March 2003. doi: 10.2337/diabetes.52.3.588. URL http://diabetes. diabetesjournals.org/cgi/doi/10.2337/diabetes.52.3.588.

[119] Barrett S D, Biwersi C, Kaufman M, Tecle H, and Warmus J S. Preparation of oxygenated esters of 4-iodophenylaminobenzhydroxyamic acids as MEK inhibitors. World Pat, April 2002.

[120] Gareth J Inman, Francisco J Nicolás, James F Callahan, John D Harling, Laramie M Gaster, Alastair D Reith, Nicholas J Laping, and Caroline S Hill. SB-431542 is a potent and specific inhibitor of transforming growth factor-beta superfamily type I activin receptor-like kinase (ALK) receptors ALK4, ALK5, and ALK7. Mol. Pharmacol., 62(1):65-74, July 2002. URL http://eutils.ncbi.nlm.nih.gov/entrez/ eutils/elink. fcgi?dbfrom=pubmed\&id=12065756\&retmode=ref\&cmd=prlinks.

[121] M Diana Neely, Michael J Litt, Andrew M Tidball, Gary G Li, Asad A Aboud, Corey R Hopkins, Reed Chamberlin, Charles C Hong, Kevin C Ess, and Aaron B Bowman. DMH1, a highly selective small molecule BMP inhibitor promotes neurogenesis of hiPSCs: comparison of PAX6 and SOX1 expression during neural induction. ACS Chem Neurosci, 3(6):482-491, June 2012. doi: 10.1021/cn300029t. URL http://eutils.ncbi.nlm.nih.gov/entrez/eutils/elink.fcgi?dbfrom=pubmed\&id=22860217\& retmode=ref\&cmd=prlinks.

[122] Jijun Hao, Joshua N Ho, Jana A Lewis, Kaleh A Karim, R Nathan Daniels, Patrick R Gentry, Corey R Hopkins, Craig W Lindsley, and Charles C Hong. In vivo structure-activity relationship study of dorsomorphin analogues identifies selective VEGF and BMP inhibitors. ACS Chem. Biol., 5(2):245-253, February 2010. doi: 10.1021/cb9002865. URL http://eutils.ncbi.nlm.nih.gov/entrez/eutils/elink.fcgi? dbfrom=pubmed\&id=20020776\&retmode=ref\&cmd=prlinks. 


\section{Supplemental Material}

\subsection{Generation of Spry4 reporter ES cell line}

The Spry4 reporter construct was generated by combining 'knock-out first' targeting arms of the EUCOMM project [24] with a H2B-Venus reporter cassette and a neomycin resistance gene driven from a human $\beta$-actin promoter. This construct was integrated by homologous recombination into a cell line carrying a doxycyclineinducible Gata4-mCherry construct described in [25]. The construct used to obtain permanent expression of the H2B-Cerulean marker has been described [25]. To generate reporter lines, approximately $2 \times 10^{6}$ cells were electroporated with $2 \mu \mathrm{g}$ of the linearised targeting vector, plated onto feeder cells and put under selection one day after electroporation. Resistant colonies were picked after seven days, and PCR-genotyped for correct insertion events (reporter constructs) or visually screened for transgene expression. Karyotypes of the Spry 4 reporter lines with the permanent nuclear marker were determined according to standard procedures [117], and clones with a median chromosome count of 40 were selected for experiments. Spry4::H2B-Venus reporter ESCs gave high-contribution chimaeras with germline transmission, confirming that this ES cell line retains full developmental potential. 
bioRxiv preprint doi: https://doi.org/10.1101/051722; this version posted May 13, 2016. The copyright holder for this preprint (which was not certified by peer review) is the author/funder, who has granted bioRxiv a license to display the preprint in perpetuity. It is made available under aCC-BY 4.0 International license.

\subsection{Supplementary Figures}

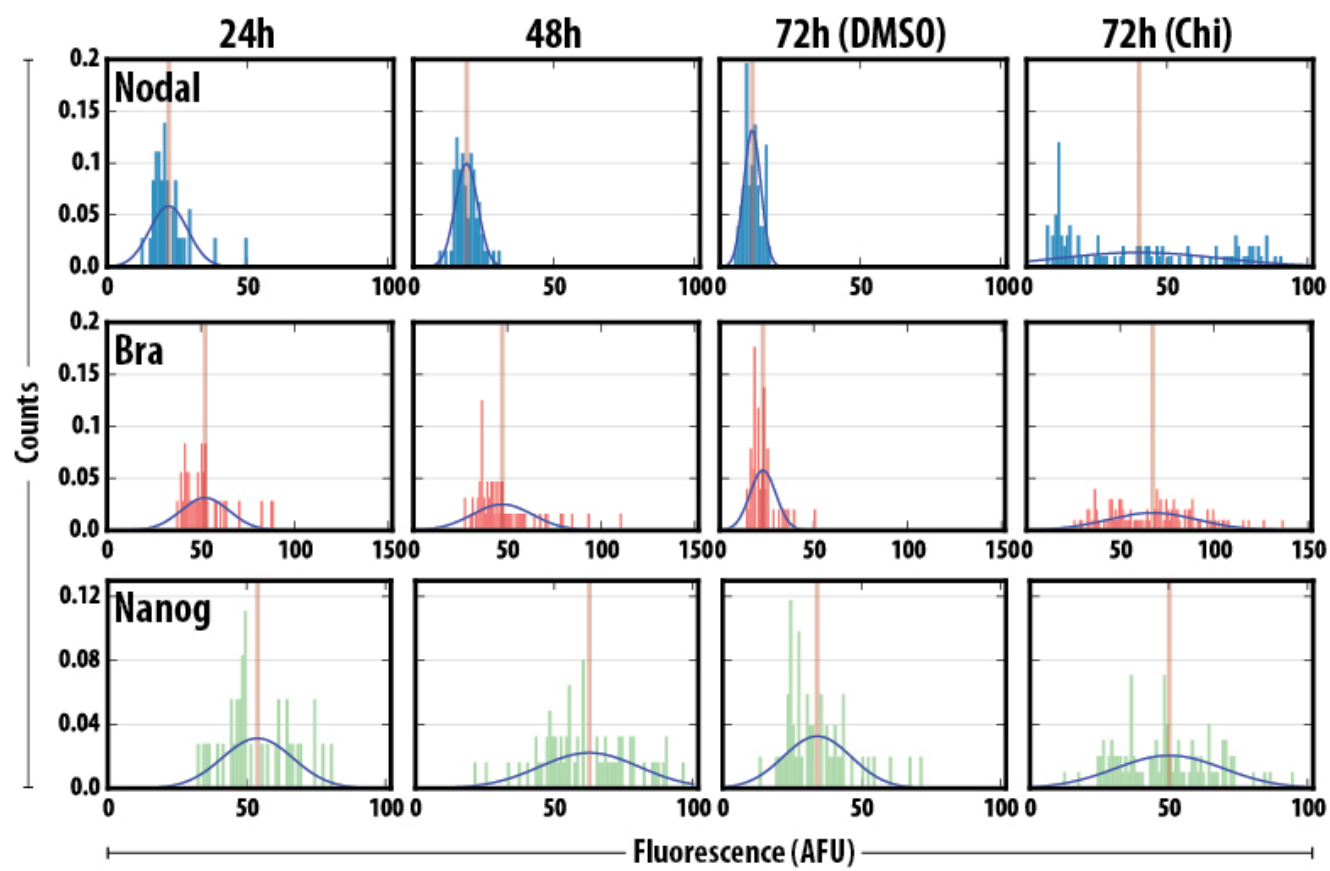

Supplementary Figure 1: Quantification of T/Bra and Nanog expression in Nodal ${ }^{\text {condHBE::YFP }}$ ESCs. A single $z$-plane from the confocal images of Gastruloids from Fig. 4 was quantified and the distributions of YFP (Blue), T/Bra (Red) and Nanog (Green) plotted for the different treatments. The horizontal orange lines in each histogram correspond to the mean fluorescence levels. 
bioRxiv preprint doi: https://doi.org/10.1101/051722; this version posted May 13, 2016. The copyright holder for this preprint (which was not certified by peer review) is the author/funder, who has granted bioRxiv a license to display the preprint in perpetuity. It is made available under aCC-BY 4.0 International license.

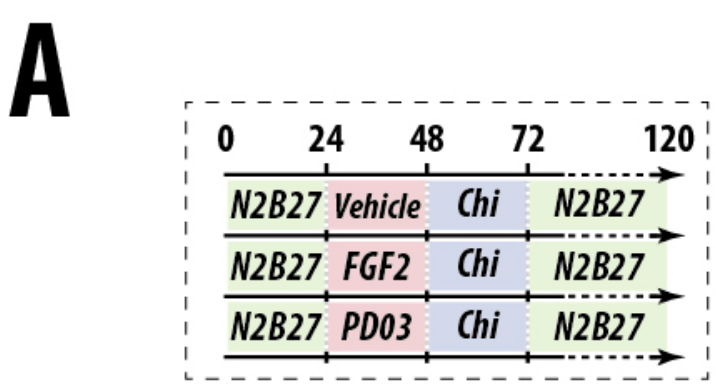

B

Pre-Pulse

Post-Pulse

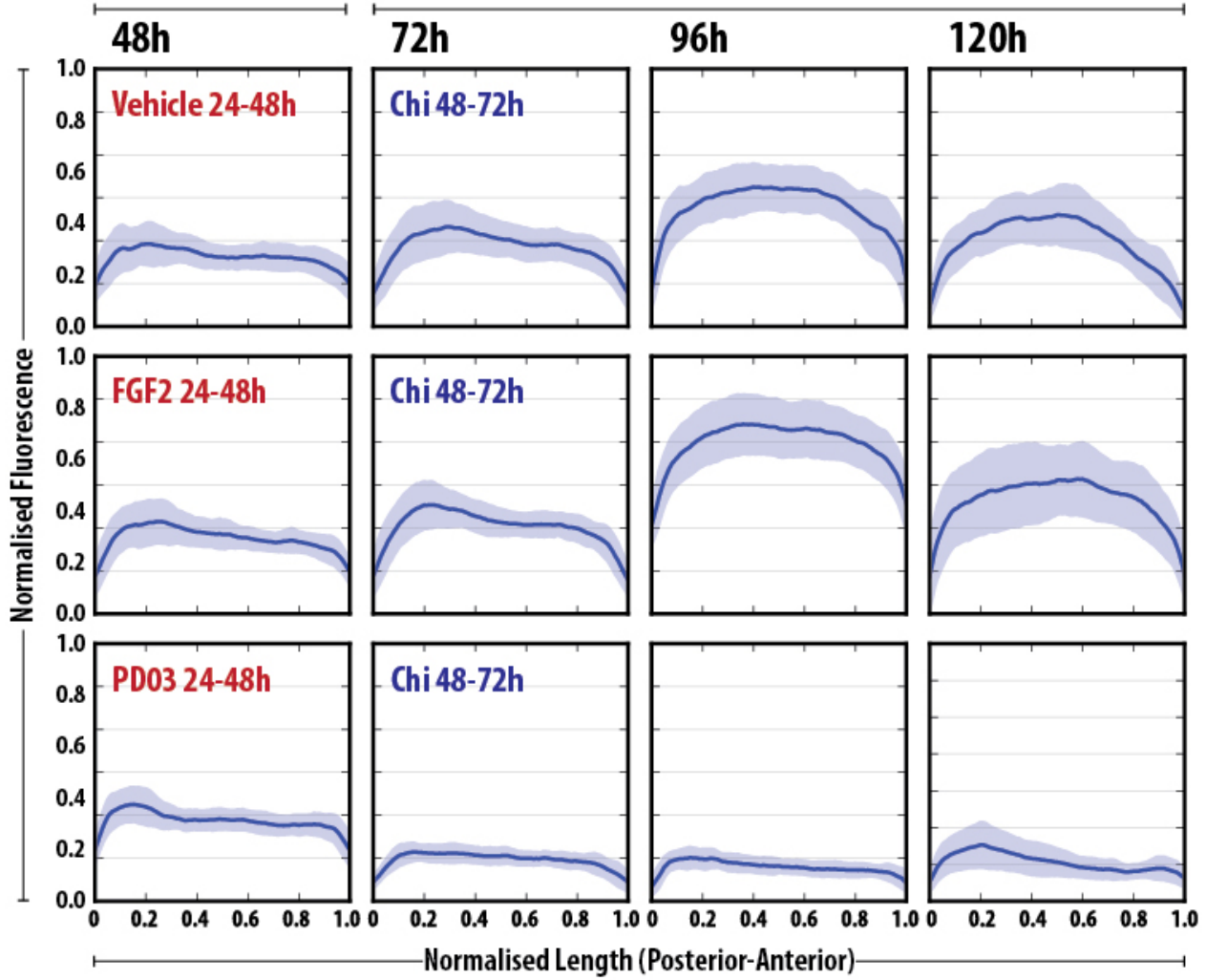

Supplementary Figure 2: The activity of the Spry4::H2B-Venus reporter in Gastruloids. (A) Schematic representation of the experimental design. Gastruloids were aggregated in N2B27 (green shading) and stimulated pulsed with Chi between 48 and 72h AA following pre-treatment vehicle, FGF2 or PD03 between 24 and $48 \mathrm{~h}$ AA. (B) The fluorescence levels of individual Spry4::H2B-Venus Gastruloids pre-treated as indicated (red text) were imaged by wide-field microscopy and quantitatively analysed for their fluorescence. The fluorescence plots were normalised to the maximum florescence of the control and the length of each Gastruloid was rescaled to have a maximum length of 1 unit. 
bioRxiv preprint doi: https://doi org/10.1101/051722; this version posted May 13, 2016. The copyright holder for this preprint (which was not certified by peer review) is the author/funder, who has granted bioRxiv a license to display the preprint in perpetuity. It is made available under aCC-BY 4.0 International license.

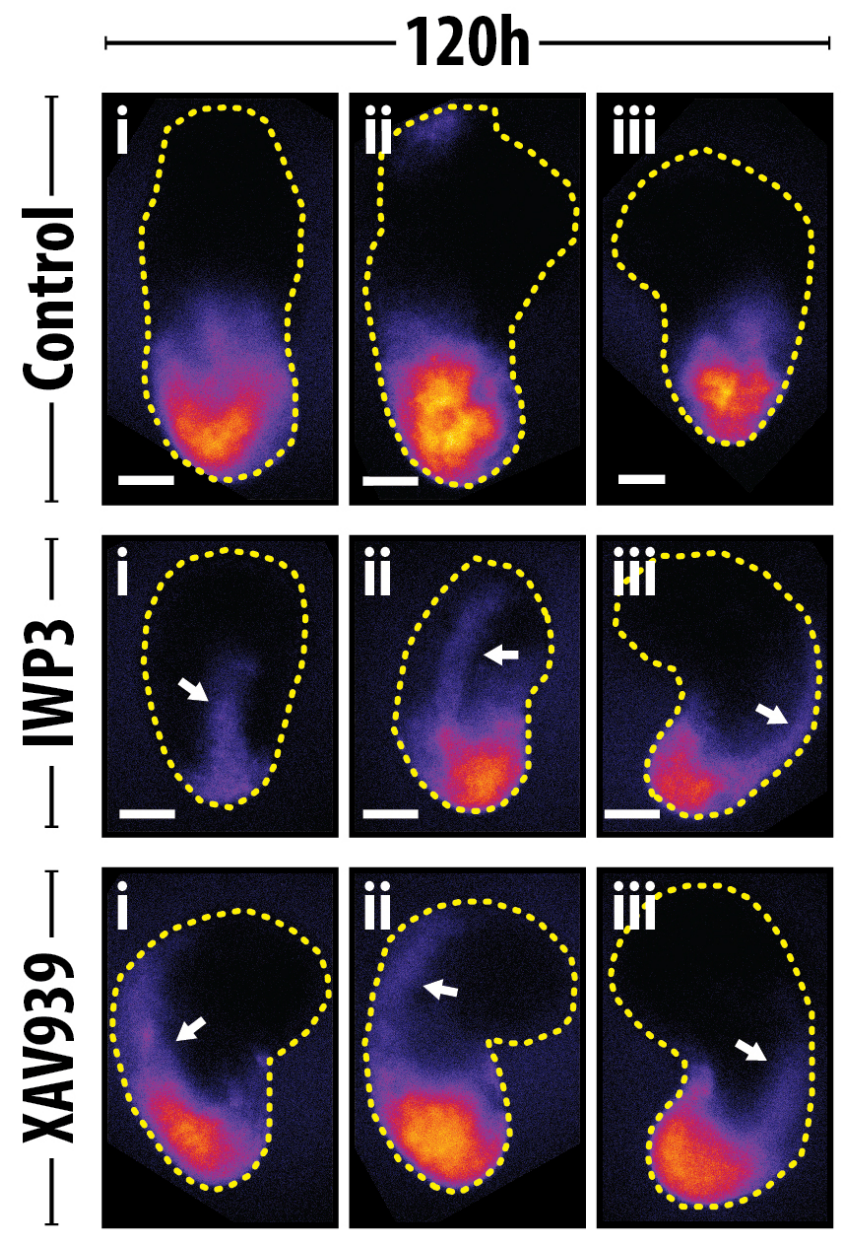

Supplementary Figure 3: Inhibition of Wnt signalling prior to the Chi pulse results in midline expression in T/Bra::GFP Gastruloids. Gastruloids formed from T/Bra::GFP mouse ESCs were pre-treated with either DMSO (vehicle), IWP3 or XAV939 for 24h before wash-off and stimulation with Chi (see Fig. 9A for experimental set up); the 120h AA time-point is indicated. Notice the extended expression of T/Bra::GFP along the midline of the Gastruloids pre-treated with either IWP3 or XAV939 (indicated by white arrows). Three examples for each condition are shown. Scale bar represents $100 \mu \mathrm{m}$. 


\subsection{Supplemental Tables}

\begin{tabular}{lllccl}
\hline & & Species & Dilution & Cat. Number & Supplier \\
\hline \multirow{3}{*}{ Primary } & Brachyury & Goat & $1: 200$ & sc-17743 & Santa Cruz Biotechnologies \\
& Nanog & Mouse & $1: 300$ & $14-5761-80$ & e-Biosciences \\
& GFP & Rabbit & $1: 500$ & A11122 & Molecular Probes \\
\hline \multirow{3}{*}{ Secondary } & Goat-A633 & Donkey & $1: 500$ & A21082 & Molecular Probes \\
& Mouse-A568 & Donkey & $1: 500$ & A10037 & Molecular Probes \\
& Rabbit-A488 & Donkey & $1: 500$ & A21206 & Molecular Probes \\
\hline & Hoechst3342 & n/a & $1: 1000$ & H3570 & Invitrogen (ThermoFisher) \\
\hline
\end{tabular}

Table 1: Antibodies used for Gastruloid immunostaining.

\begin{tabular}{llcl}
\hline Cell Line & & Cells $/ 40 \mu \mathrm{l}$ & 48h Diameter $(\mu \mathrm{m})$ \\
\hline AR8::mCherry & {$[18]$} & 550 & $182.7 \pm 17.3(n=83)$ \\
Bra::GFP & {$[16]$} & 400 & $161.0 \pm 26.2(n=222)$ \\
IBRE4::mCerulean & {$[18]$} & 400 & $152.6 \pm 12.2(n=39)$ \\
Nodal $^{\text {condHBE::YFP }}$ & {$[17]$} & 500 & $138.7 \pm 16.1(n=124)$ \\
Spry4::VNP & This work & 550 & $179.4 \pm 20.3(n=53)$ \\
TLC2 & {$[19,20]$} & 400 & $194.9 \pm 20.7(n=56)$ \\
\hline
\end{tabular}

Table 2: Cell lines used and numbers of cells required for Gastruloid culture. The average diameter of the Gastruloids at $48 \mathrm{~h}$ post formation is indicated with the standard deviation and the number of Gastruloids measured from at least two replicate experiments.

\begin{tabular}{lllll}
\hline $\begin{array}{l}\text { Small Molecule/ } \\
\text { Recombinant protein }\end{array}$ & & Working Conc. & Stock Conc. & \multirow{2}{*}{ Supplier } \\
\hline CHI99201 & {$[118]$} & $3 \mu \mathrm{M}$ & $10 \mathrm{mM}$ & \\
PD0325901 & {$[119]$} & $1 \mu \mathrm{M}$ & $10 \mathrm{mM}$ & \\
SB431542 & {$[120]$} & $10 \mu \mathrm{M}$ & $100 \mathrm{mM}$ & \multirow{2}{*}{ Tocris Biosciences } \\
DMH1 & {$[121,122]$} & $0.5 \mathrm{nM}$ & $5 \mathrm{mM}$ & \\
IWP3 & {$[35]$} & $1 \mu \mathrm{M}$ & $5 \mathrm{mM}$ & \\
XAV939 & {$[36]$} & $1 \mu \mathrm{M}$ & $10 \mathrm{mM}$ & \\
\hline Wnt3a & & $100 \mathrm{ng} / \mathrm{ml}$ & $40 \mu \mathrm{g} / \mathrm{ml}$ & \\
DKK & & $200 \mathrm{ng} / \mathrm{ml}$ & $100 \mu \mathrm{g} / \mathrm{ml}$ & \\
Nodal & $1 \mu \mathrm{g} / \mathrm{ml}$ & $50 \mu \mathrm{g} / \mathrm{ml}$ & \multirow{2}{*}{ R\&D systems } \\
Noggin & & $500 \mathrm{ng} / \mathrm{ml}$ & $100 \mu \mathrm{g} / \mathrm{ml}$ & \\
Chordin & & $500 \mathrm{ng} / \mathrm{ml}$ & $200 \mu \mathrm{g} / \mathrm{ml}$ & \\
FGF2 & & $10 \mathrm{ng} / \mathrm{ml}$ & $100 \mu \mathrm{g} / \mathrm{ml}$ & \\
\hline
\end{tabular}

Table 3: Concentrations of Small molecules and recombinant proteins used in this study. Conc: Concentration 


\begin{tabular}{lll}
\hline Gene & Forward Primer Sequence & Reverse Primer Sequence \\
\hline Axin2 & CTAGACTACGGCCATCAGGAA & GCTGGCAGACAGGACATACA \\
Bmp 4 & CTCAAGGGAGTGGAGATTGG & ATGCTTGGGACTACGTTTGG \\
Cer1 & GGAAACGCCATAAGTCTCCA & AGGGTCAGAATTTGCCATTG \\
Chordin & GTGCCTCTGCTCTGCTTCTT & AGGAGTTCGCATGGATATGG \\
Dkk1 & CCATTCTGGCCAACTCTTTC & CATTCCCTCCCTTCCAATAAC \\
Fgf 4 & GGCCACTCCACAGAGATAGG & ACTTGGGCTCAAGCAGTAGG \\
Fgf5 & GCTCAATGATCAGAAGGAGGA & TCAGCTGGTCTTGAATGAGG \\
Fgfs & AGGACTGCGTATTCACAGAGAT & CATGTACCAGCCCTCGTACT \\
Lefty1 & AGGGTGCAGACCTGTAGCTG & GGAAGCAAAGAGCACACACA \\
Nodal & AGCCACTGTCCAGTTCTCCAG & GTGTCTGCCAAGCATACATCTC \\
Noggin & CCCATCATTTCCGAGTGTAAG & CTCGCTAGAGGGTGGTGAAA \\
ppia & TTACCCATCAAACCATTCCTTCTG & AACCCAAAGAACTTCAGTGAGAGC \\
SPRY4 & ATGGTGGATGTCGATCCTGT & GGAGGGGGAGCTACAGAGAC \\
T/Bra & CTGGGAGCTCAGTTCTTTCG & GTCCACGAGGCTATGAGGAG \\
Wnt3 & CTAATGCTGGCTTGACGAGG & ACATGGTAGAGAGTGCAGGC \\
Wnt $3 a$ & CATACAGGAGTGTGCCTGGA & AATCCAGTGGTGGGTGGATA \\
\hline
\end{tabular}

Table 4: Primer Sequences used for qRT-PCR.

\begin{tabular}{lllll||llll} 
& Condition & No Expression & Polarised & Ubiquitous & Spherical & Ovoid & Elongated & $n$ \\
\hline 24h & \multirow{2}{*}{ N2B27 } & $26.8(21.5)$ & $62.5(16.1)$ & $10.7(15.2)$ & $100.0(0.0)$ & $0.0(0.0)$ & $0.0(0.0)$ & 112 \\
$48 \mathrm{~h}$ & & $23.7(13.2)$ & $74.1(11.8)$ & $2.2(3.4)$ & $67.0(9.4)$ & $33.0(9.4)$ & $0.0(0.0)$ & 140 \\
\hline \multirow{2}{*}{$72 \mathrm{~h}$} & N2B27 & $6.5(6.6)$ & $89.4(8.5)$ & $4.1(4.4)$ & $25.7(16.4)$ & $55.5(24.7)$ & $18.8(14.7)$ & 89 \\
& DMSO & $3.6(-)$ & $89.3(-)$ & $7.1(-)$ & $10.7(-)$ & $85.7(-)$ & $3.6(-)$ & 28 \\
& Chi & $0(-)$ & $91.2(11.7)$ & $8.8(11.7)$ & $23.3(18.2)$ & $52.9(18.1)$ & $23.8(26.3)$ & 82 \\
\hline
\end{tabular}

Table 5: Expression phenotype of T/Bra::GFP mouse ESCs. The proportion of T/Bra::GFP Gastruloids not expressing the reporter (No Expression) or displaying either Polarised or Ubiquitous expression at 24, 48 and $72 \mathrm{~h}$ AA when maintained in N2B27 $(24,48,72 \mathrm{~h})$ or following a pulse of DMSO or Chi $(72 \mathrm{~h})$. The standard deviation is shown in brackets and the number of Gastruloids analysed are shown $(n)$.

\begin{tabular}{lll}
\hline \multicolumn{3}{c}{ Pearson Correlation Coefficient } \\
\hline $24 \mathrm{~h} \quad 48 \mathrm{~h} \quad \mathrm{~N} 2 \mathrm{~B} 2772 \mathrm{~h} \quad$ Chi $72 \mathrm{~h}$
\end{tabular}

\begin{tabular}{lcccc}
\hline a) Nodal:Bra & -0.029 & 0.592 & 0.807 & 0.643 \\
b) Nodal:Nanog & 0.112 & 0.452 & 0.814 & 0.280 \\
c) T/Bra:Nanog & 0.391 & 0.443 & 0.731 & 0.327 \\
\hline
\end{tabular}

Table 6: Pearson Correlation Coefficients between a) Nodal and Brachyury, b) Nodal and Nanog and c) Brachyury and Nanog in the Nodal ${ }^{\text {condH BE::YFP }}$ mouse ESC line at 24,48 and $72 \mathrm{~h}$ with or without a pulse of Chi on day 3. Values to 3d.p., correlations from one representative Gastruloid 